\title{
Mild impairment of mitochondrial function increases longevity and pathogen resistance through ATFS-1-driven activation of p38-regulated innate immunity
}

\author{
Juliane C. Campos ${ }^{1,2,3,4 \#}$, Ziyun $\mathrm{Wu}^{1,2,3 \#}$, Paige D. Rudich ${ }^{5,6}$, Sonja K. Soo ${ }^{5,6}$, Meeta Mistry ${ }^{7}$, \\ Julio C.B. Ferreira ${ }^{4}$, T. Keith Blackwell ${ }^{1,2,3^{*}}$, Jeremy M. Van Raamsdonk ${ }^{2,5,6,8^{*}}$ \\ ${ }^{1}$ Research Division, Joslin Diabetes Center, Boston, MA, USA \\ ${ }^{2}$ Department of Genetics, Harvard Medical School, Boston, MA, USA \\ ${ }^{3}$ Harvard Stem Cell Institute, Harvard Medical School, Boston, MA, USA \\ ${ }^{4}$ Institute of Biomedical Sciences, University of Sao Paulo, Sao Paulo, Brazil \\ ${ }^{5}$ Department of Neurology and Neurosurgery, McGill University, Montreal, Quebec, Canada \\ ${ }^{6}$ Metabolic Disorders and Complications Program, and Brain Repair and Integrative \\ Neuroscience Program, Research Institute of the McGill University Health Centre, Montreal, \\ Quebec, Canada \\ ${ }^{7}$ Bioinformatics Core, Harvard School of Public Health, Harvard Medical School, Boston. MA, \\ USA \\ ${ }^{8}$ Division of Experimental Medicine, Department of Medicine, McGill University, Montreal, \\ Quebec, Canada
}

\#These authors contributed equally to this work

*These authors are co-corresponding authors

Short title: Mitochondrial function, innate immunity, and aging

\section{Corresponding Authors:}

T. Keith Blackwell

Joslin Diabetes Center

1 Joslin Place, Boston, MA, USA

1-617-309-1981, keith.blackwell@joslin.harvard.edu

Jeremy M. Van Raamsdonk

Research Institute of the McGill University Health Centre

1001 Decarie Blvd., Montreal, Quebec, Canada

1-514-934-1934 ext.76157, jeremy.vanraamsdonk@mcgill.ca 


\section{Abstract}

3 While mitochondrial function is essential for life in all multicellular organisms, a mild

$4 \quad$ impairment of mitochondrial function can extend longevity. By understanding the molecular

5 mechanisms involved, these pathways might be targeted to promote healthy aging. In studying

6 two long-lived mitochondrial mutants in C. elegans, we found that disrupting subunits of the

7 mitochondrial electron transport chain resulted in upregulation of genes involved in innate

8 immunity, which we found to be dependent on not only the canonical p38-mediated innate

9 immune signaling pathway but also on the mitochondrial unfolded protein response. Both of

10 these pathways are absolutely required for the increased resistance to bacterial pathogens and

11 extended longevity of the long-lived mitochondrial mutants, as is the FOXO transcription factor

12 DAF-16. This work demonstrates that both the p38-mediated innate immune signaling pathway

13 and the mitochondrial unfolded protein response can act on the same innate immunity genes

14 to promote resistance to bacterial pathogens, and that input from the mitochondria can extend

15 longevity by signaling through these two pathways. Combined, this indicates that multiple

16 evolutionarily conserved genetic pathways controlling innate immunity also function to

17 modulate lifespan.

19 Keywords: Aging; Innate Immunity; C. elegans; Mitochondria; Mitochondrial unfolded protein

20 response; Genetics; Lifespan; ATFS-1; p38/PMK-1 


\section{Significance Statement}

22

23 In this work, we explore the relationship between mitochondrial function, aging and innate

24 immunity. We find that mild impairment of mitochondrial function results in upregulation of

25 genes involved in innate immunity, increased resistance to bacterial pathogens and lifespan

26 extension, all of which are dependent on two evolutionarily conserved signaling pathways. This

27 work demonstrates how changes in functional status of the mitochondria can trigger activation

28 of innate immunity, and that the underlying mechanisms are important for the longevity of the

29 organism. This work advances our understanding of connections between metabolism and

30 immunity. As the pathways studied here are conserved up to mammals, these insights may help

31 us to understand the role of mitochondrial health, innate immunity and lifespan in humans. 


\section{Introduction}

While aging was long believed to be a stochastic process of damage accumulation, research during the past three decades has demonstrated that lifespan can be strongly influenced by genetics. Single-gene mutations have been shown to extend longevity in model organisms, including yeast, worms, flies and mice. Importantly, genes and interventions that increase lifespan tend to be conserved across species. For example, decreasing insulin-IGF1 signaling, which was first shown to increase lifespan in the worm C. elegans(1-3), has subsequently been shown to extend longevity in flies(4) and mice(5), and to be associated with longevity in humans(6). This suggests that studying the aging process in model organisms can provide insights that are relevant to human aging.

The first single gene mutation that was shown to extend lifespan was identified in C. elegans(2, 3), and since then this organism has been extensively used to find additional genetic pathways associated with lifespan extension and to elucidate the underlying mechanisms. Among the earliest genes that were shown to influence longevity were genes involved in mitochondrial function. Mutations in $c k-1$, nuo- 6 and isp-1 affect different components of the mitochondrial electron transport chain, and all lead to increased lifespan(7-10). In the case of nuo-6 and isp-1, which encode subunits of complex I and complex III respectively, a complete loss of function mutation would be lethal, while point mutations that result in a mild impairment of mitochondrial function extend longevity. Similarly, decreasing the expression of genes involved in mitochondrial function with RNA interference also increases lifespan(11, 12). Importantly, mutations that affect mitochondrial function have also been shown to increase lifespan in other species, including flies(13) and mice $(14,15)$.

While initially it was believed that the mechanism by which mild impairment of mitochondrial function increased lifespan was through a decrease in the production of reactive oxygen species (ROS) and the resulting oxidative damage(9), more recent studies show that mutations affecting 
61 for the long lifespan of these mutants, as treatment with antioxidants can decrease their

62 lifespan to wild-type $(16,17)$. While multiple factors contributing to the long lifespan of these

63 mitochondrial mutants have been identified(18-23), the precise mechanisms of lifespan

64 extension remain incompletely understood. In our previous work, we have shown that two stress-responsive transcription factors, DAF-16/FOXO3 and ATFS-1/ATF5, are required for the long-lifespan of nuo-6 and isp-1 worms $(19,20)$.

DAF-16/FOXO3 is a FOXO transcription factor that is directly regulated by phosphorylation in response to insulin-IGF1 signaling, a growth factor signaling pathway that begins with the insulin-IGF1 receptor DAF-2. While DAF-16 normally resides in the cytoplasm, when signaling through the insulin-IGF1 pathway is reduced DAF-16 accumulates in nuclei. DAF-16 also translocates to the nucleus in response to various stresses. In the nucleus, DAF-16 upregulates genes involved in stress response and metabolism(24, 25).

Activating transcription factor associated with stress 1 (ATFS-1/ATF5) is the transcription factor that mediates the mitochondrial unfolded protein response (mitoUPR)(26), a stress response pathway that responds to mitochondrial stress in order to restore mitochondrial function. ATFS-1 contains a nuclear localization signal (NLS) and a mitochondrial targeting sequence (MTS). Under normal conditions, ATFS-1 is targeted to the mitochondria where it is imported and degraded. Under conditions of mitochondrial stress, mitochondrial import of ATFS-1 is prevented, resulting in accumulation of ATFS-1 in the cytoplasm, where the NLS translocates ATFS-1 into the nucleus in order to restore mitochondrial homeostasis through alterations in expression of genes involved in protein folding and metabolism(27).

While DAF-16 and ATFS-1 can both contribute to defense against bacterial pathogens $(28,29)$, the primary innate immune signaling pathway that responds to bacterial pathogen stress is a mitogen-activated protein kinase (MAPK) signaling pathway, which has been found to be conserved from invertebrates to mammals(30-32). In this pathway, NSY-1/ASK1 (MAPK kinase kinase) signals to SEK-1/MKK3/MKK6 (MAPK kinase), which signals to PMK-1/p38 (MAPK) (33, 
34) (Fig. S1). Downstream of this pathway, the transcription factor ATF-7/ATF2/ATF7/CREB5 acts to modulate the expression of genes involved in innate immunity $(35,36)$. While ATF-7 normally acts as a repressor of gene function, when it is phosphorylated by PMK-1, ATF-7 functions as an activator of p38/ATF-7-regulated immunity gene expression (Fig. S1).

In this work, we show that the p38-mediated innate immune signaling pathway is essential for the longevity and pathogen resistance of the long-lived mitochondrial mutants, nuo- 6 and isp-1.

97 We find that both strains exhibit an upregulation of genes involved in innate immunity that is

98 driven by the activation of the mitoUPR, but also dependent on the p38-mediated innate

99 immune signaling pathway, leading to an increased resistance to bacterial pathogens. The p38-

100 mediated innate immune signaling pathway is absolutely required for the long lifespan of nuo-6

101 and isp-1 mutants. Finally, we demonstrate that activation of the mitoUPR is sufficient to

102 upregulate innate immunity genes, and is also required for their upregulation in nuo-6 mutants.

103 Overall, this work demonstrates the importance of the mitoUPR in upregulating innate

104 immunity in response to signals from the mitochondria, and delineates a clear role of innate 105 immune signaling pathways in determining lifespan. 


\section{Results}

\section{Long-lived mitochondrial mutants exhibit broad upregulation of genes involved in innate} immunity that is dependent on p38-mediated innate immune signaling pathway

While mild impairment of mitochondrial function has been shown to extend longevity, the underlying mechanisms are yet to be fully elucidated. When mitochondrial function is impaired, mitochondria are able to communicate with the nucleus to alter nuclear gene expression. To obtain a comprehensive, unbiased view of the transcriptional changes that result from impairment of mitochondrial function, we used RNA sequencing (RNA-seq) to examine gene expression in two long-lived mitochondrial mutants, nuo-6 and isp-1. After determining which genes were differentially expressed compared to wild-type worms, we identified groups of genes that showed enrichment. Among the genes that showed enrichment were genes involved in innate immunity. These genes encode proteins that function to inhibit the growth and survival of pathogenic bacteria, and to repair or remove damage to the worm $(37,38)$.

Accordingly, we decided to investigate the role of innate immunity in the long lifespan of these long-lived mitochondrial mutants.

To determine the extent to which genes involved in innate immunity are upregulated in the long-lived mitochondrial mutants, nuo- 6 and isp-1, we first examined eight genes, which others have used to monitor innate immune activity $(29,35,37,39-41)$. These genes included T24B8.5, K08D8.5, F55G11.8, clec-65, clec-67, dod-22, Y9C9A.8 and C32H11.4. All of these genes are upregulated in response to exposure to the bacterial pathogen Pseudomonas aeruginosa strain PA14 and five of eight have been shown to be direct targets of the p38-mediated innate immune signaling pathway in a ChIP-seq analysis of ATF-7(36). In examining the expression of these genes in our RNA-seq data, we found that all eight genes were significantly upregulated in both nuo-6 and isp-1 worms (Fig. 1A). 
134 To determine whether the upregulation of innate immunity genes in the long-lived mitochondrial mutants requires the p38-mediated innate immune signaling pathway (NSY-1 $\rightarrow$ SEK-1 $\rightarrow$ PMK-1 $\rightarrow$ ATF-7), we generated double mutants of nuo- 6 and isp- 1 with all of these genes before measuring gene expression with quantitative RT-PCR (qPCR). We used loss of function mutants for $n s y-1$, sek-1 and pmk-1. Since ATF-7 normally acts as a repressor, we used the $q d 22$ gain-of-function mutation for this gene. This mutation prevents phosphorylation of ATF-7 by PMK-1 thereby making the mutant ATF-7 a constitutive repressor(35) (Fig. S1). As in the RNA-seq data, the results from the qPCR experiments showed that innate immune genes are upregulated in nuo- 6 and isp-1 worms. Importantly, we found that in both nuo- 6 and isp-1 worms that disruption of genes involved in the p38-mediated innate immune signaling pathway (nsy-1, sek-1, pmk-1, atf-7(gof)) prevented the upregulation of innate immunity genes (Fig. 1B, Fig. S2).

To confirm these results using an alternative approach, we crossed a fluorescent reporter strain for one of the innate immunity genes (T24B8.5)(35) to nuo- 6 and isp-1 mutants and examined the effect of knocking down sek-1 through RNA interference (RNAi). Again, using this approach, we found that T24B8.5 is upregulated in nuo- 6 and isp- 1 worms, and that this upregulation is dependent on SEK-1 (Fig. 1C). Combined, these results demonstrate that innate immunity genes are upregulated in the long-lived mitochondrial mutants, nuo- 6 and isp-1, and that this upregulation is dependent on the p38-mediated innate immune signaling pathway.

To further examine the expression of innate immunity genes in nuo- 6 and isp-1 mutants, we compared the differentially expressed genes in these mutants to a more comprehensive and unbiased list of genes involved in innate immunity. A recent study defined the changes in gene expression that result from exposure to the bacterial pathogen Pseudomonas aeruginosa strain PA14 and determined which of these changes in gene expression are dependent on PMK-1 and ATF-7(36). In total, they reported 300 genes that were upregulated by exposure to PA14 in a PMK-1 and ATF-7-dependent manner, and 230 genes that were downregulated by exposure to PA14 in a PMK-1 and ATF-7-dependent manner. 
164 We compared these lists of PA14-modulated, PMK-1-dependent, ATF-7-dependent genes to

165 genes that we found to be significantly upregulated or downregulated in nuo-6 and isp-1

166

167

168

169

170

171

172

173

174

175

176

177

178

179

180

181

182

183

184

185

186

187

188

189

190

191

mutants. We found that of the genes that are upregulated by PA14 exposure in a PMK-1 and ATF-7-dependent manner, $38 \%\left(p=1.5 \times 10^{-35}\right)$ and $30 \%\left(p=3.7 \times 10^{-27}\right)$ are also upregulated in nuo-6 and isp-1 mutants, respectively (Fig. 1D). There is a high degree of overlap between genes upregulated in nuo- 6 and isp-1 mutants (71\%), and this includes the genes involved in innate immunity (Table S1; 71 overlapping genes of 89/115 innate immunity genes upregulated in nuo-6 or isp-1, respectively). In contrast, there was no significant overlap of the same genes upregulated by PA14 exposure with genes downregulated in nuo- 6 and isp-1 mutants. In examining the list of genes that are downregulated by exposure to PA14 in a PMK-1 and ATF-7dependent manner, we found that $20 \%\left(p=8.4 \times 10^{-7}\right)$ and $20 \%\left(p=7.5 \times 10^{-5}\right)$ were also downregulated in nuo- 6 and isp-1 mutants, respectively.

To more comprehensively compare the PA14-modulated, PMK-1-dependent, ATF-7-dependent gene expression changes to gene expression changes in nuo- 6 and isp-1 mutants, we generated heat maps comparing the expression of PA14-modulated, PMK-1-dependent, ATF-7-dependent genes between wild-type worms and nuo-6 or isp-1 mutants. Among the genes that are upregulated by PA14 exposure in a PMK-1 and ATF-7-dependent manner, many of these genes are upregulated in nuo-6 and isp-1 mutants compared to wild-type worms, while a small subset show decreased expression (Figs. S3,S4). Among the genes that are downregulated by PA14 exposure in a PMK-1 and ATF-7-dependent manner, many of these genes are downregulated in nuo- 6 and isp-1 mutants compared to wild-type worms, while a number of these genes also show increased expression (Figs. S5,S6).

\section{Overall, these results indicate that a mild impairment of mitochondrial function caused by} mutations in nuo- 6 or isp-1 leads to a broad upregulation of genes involved in innate immunity that is dependent on the p38-mediated innate immune signaling pathway. At the same time, a smaller proportion of these genes show decreased expression suggesting that the correct 
balance of innate immune gene expression may be required to optimize stress resistance and lifespan.

\section{Long-lived mitochondrial mutants have increased resistance to bacterial pathogens, which} requires the p38-mediated innate immune signaling pathway

Based on our observation that the long-lived mitochondrial mutants, nuo- 6 and isp-1, have increased expression of many genes involved in innate immunity, we next sought to determine whether this increase in their expression resulted in enhanced resistance to bacterial pathogens. To test pathogen resistance, we exposed worms to PA14 in a slow kill assay where worms die from the ingestion and internal proliferation of the PA14 bacteria(42-44). We found that both nuo- 6 and isp-1 worms exhibited significantly increased survival on PA14 bacteria compared to wild-type worms (Fig. 2A). The increase in survival of nuo- 6 and isp-1 worms did not result from reduced exposure to the pathogenic bacteria as their tendency to avoid PA14 was equivalent to wild-type worms (Fig. S7).

To determine the extent to which their enhanced resistance to bacterial pathogens is dependent on the p38-mediated innate immune signaling pathway, we next examined PA14 resistance in nuo-6 and isp-1 worms in which genes in this pathway were disrupted. In wild-type worms, mutations in nsy-1, sek-1, pmk-1 or atf-7(gof) significantly decrease the survival of worms exposed to PA14 (Fig. 2B). Similarly, we found that disruptions of these innate immune signaling genes in nuo-6 (Fig. 2C) or isp-1 (Fig. 2D) worms also results in a significant decrease in survival on PA14 bacteria. In nuo-6 worms, survival was decreased back to wild-type by mutations in nsy-1, pmk-1 or atf-7(gof), while a larger decrease in survival was observed with the sek-1 mutation. In isp-1 worms, mutations in nsy-1, sek-1 and atf-7(gof) all decreased survival to a greater extent than in nuo- 6 mutants, and in each case the survival of the double mutant was less than in wild-type worms (Fig. S8). Combined, this shows that nuo- 6 and isp-1 worms have increased resistance to bacterial pathogens, which is dependent on the p38mediated innate immune signaling pathway. 
p38-mediated innate immune signaling pathway is required for extended longevity in long-

lived mitochondrial mutants

The p38-mediated innate immune signaling pathway is required for the lifespan extension resulting from decreased insulin-IGF1 signaling (daf-2 mutants) and dietary restriction $(38,39)$. Interestingly, in both daf-2 mutants and dietary restricted worms expression of p38-regulated innate immunity genes is largely decreased, and activation of this pathway to higher levels is deleterious for lifespan extension, suggesting that a lower level of immune activation is optimal under these conditions(39). We decided to examine the role of the p38-mediated innate

231 immune signaling pathway in the long lifespan of nuo-6 and isp-1 mutants, in which we found

232 these immunity genes were largely upregulated. To do this, we genetically disrupted components of the p38-mediated innate immune signaling pathway in nuo- 6 and isp-1 mutants and quantified the resulting effect on lifespan. In nuo-6 mutants, we found that mutation of nsy-1, sek-1, pmk-1 or atf-7(gof) reduced the long lifespan of nuo- 6 worms to near wild-type lifespan, almost completely preventing any increase in lifespan resulting from the nuo-6 mutation (Fig. 3A-D). Similarly, we found that mutations in the p38-mediated innate immune signaling pathway also markedly reduced the lifespan of isp-1 worms (Fig. 3E-H). Mutations in nsy-1, sek-1, or atf-7(gof) decreased isp-1 lifespan to near wild-type lifespan, while pmk-1 RNAi reduced the lifespan extension in isp-1 worms by half (we were unable to generate isp-1 pmk-1 double mutants because of the proximity of the two genes on the same chromosome).

Although OP50 bacteria, which is typically used for C. elegans maintenance and lifespan assays,

244 is considered to be non-pathogenic, it can have detrimental effects on lifespan through

245 proliferation in the pharynx and intestine $(45,46)$. Accordingly, we also measured the lifespan

246 of nuo-6 and isp-1 mutants on non-proliferating OP50 bacteria, as well as the dependency of

247 their long lifespan on the p38-mediated innate immunity pathway under these conditions.

248 Similar to what we observed on live bacteria, we found that nuo- 6 and isp-1 mutants are long- 
lived on non-proliferating bacteria, and that their long lifespan is still completely dependent on the p38-mediated innate immune signaling pathway (Fig S9).

Combined, these results clearly indicate that the p38-mediated innate immune signaling pathway is required for the extended longevity in the long-lived mitochondrial mutants, nuo- 6 and isp-1. However, the fact that effect of disrupting the p38-innate immune signaling pathway on nuo-6 and isp-1 lifespan is independent of bacterial proliferation suggests that the lifespan extension resulting from mild impairment of mitochondrial function is not necessarily a direct consequence of their enhanced resistance to bacterial pathogens, but more likely a result of other functions of the p38-mediated innate immune signaling pathway.

\section{p38-mediated innate immune signaling pathway is not activated in long-lived mitochondrial} mutants

Since p38 signaling can be activated by $\operatorname{ROS}(47,48)$, and ROS levels are increased in nuo- 6 and isp-1 mutants(16), we hypothesized that the upregulation of innate immunity genes in these mutants resulted from increased signaling through the p38-mediated innate immune signaling pathway. To test this, we examined the extent to which this pathway might be activated in these mutants by quantifying the ratio of phosphorylated (active) p38/PMK-1 to total p38/PMK1 by Western blotting(47). To validate this approach, we showed that decreasing innate immune signaling with sek-1 RNAi and increasing innate immune signaling with vhp-1 RNAi (VHP-1 is a phosphatase that inhibits PMK-1/p38 signaling) alters the ratio of phospho-p38 to p38 as expected (Fig. S10).

Having shown that known modulators of PMK-1/p38 activation alter PMK-1/p38 phosphorylation in a predictable manner, we examined PMK-1/p38 activation in nuo-6 and isp1 mutants. In both cases we found that neither the levels of PMK-1/p38 protein nor the proportion of PMK-1/p38 protein that is phosphorylated are increased compared to wild-type

(Fig. 4A,B). Thus, even though nuo-6 and isp-1 mutants have increased expression of ATF-7 
target genes, there is no detectable increase in PMK-1/p38 activation. Since the p38-mediated innate immune signaling pathway is required for the increased expression of ATF-7 target genes even though p38 signaling is not increased, this suggests that this pathway is playing a permissive role, and that other mechanisms are driving the innate immune activation in the long-lived mitochondrial mutants.

Since the p38-mediated innate immune signaling pathway can be activated by nutrient consumption independently of pathogen exposure(39), we wondered whether altered feeding affects the level of activation of this pathway in the long-lived mitochondrial mutants, countering the effects of elevated ROS. Accordingly, we quantified food consumption in nuo- 6 and isp-1 worms directly by monitoring the decrease in OP50 bacteria from day 3 to day 12 of adulthood(39). We found that both long-lived mutants show a dramatic decrease in food consumption compared to wild-type worms (Fig. 4C). This decrease in food consumption does not result from a difference in occupancy of the bacteria as nuo- 6 and isp-1 worms spend the amount of time on the bacteria as wild-type worms (Fig. S7). The decrease in food consumption in nuo-6 and isp-1 worms would be predicted to decrease p38-mediated innate immune signaling, and might be sufficient to decrease any ROS-induced activation of this pathway in these mutants. The fact that the activation of the p38-mediated innate immune signaling pathway is not decreased in the long-lived mitochondrial mutants, and that target genes of this pathway are upregulated, again indicates that other factors are acting in parallel to p38 signaling to upregulate the expression of innate immunity genes.

\section{Decreased feeding in long-lived mitochondrial mutants is mediated by activation of the} mitoUPR

We next wanted to define the mechanism by which feeding is decreased in the long-lived mitochondrial mutants. Since DAF-16 is activated in nuo-6 and isp-1 mutants (20) and disruption of daf-16 has been shown to increase food intake in wild-type and daf-2 worms(39), we sought to determine whether $d a f-16$ is required for the decreased food intake observed in 
the long-lived mitochondrial mutants. We found that deletion of daf-16 increased food consumption in both wild-type and isp-1 worms (Fig. 4D), thereby indicating that DAF-16 is required for the decreased food consumption in isp-1 mutants.

Since activation of ATFS-1 is sufficient to upregulate DAF-16 target genes(19) and ATFS-1 is activated in the long-lived mitochondrial mutants(19), we wondered whether ATFS-1 also contributes to the decreased food intake in the long-lived mitochondrial mutants. While disruption of atfs-1 (using the gk3094 deletion mutation) had no effect on feeding in a wildtype background, loss of atfs-1 reverted food consumption in nuo- 6 worms to wild-type (Fig. 4E). This indicates that both $d a f-16$ and atfs-1 are required for the decreased food intake in the long-lived mitochondrial mutants.

Having shown that activation of ATFS-1 contributes to the decreased food intake in nuo- 6 mutants, we wondered whether activation of ATFS-1 alone is sufficient to decrease food consumption. To test this idea, we measured food consumption in a constitutively activated atfs-1 mutant (et17). In this mutant, the mitochondrial targeting sequence of ATFS-1 is disrupted resulting in nuclear localization and constitutive activation of ATFS-1 target genes(49). We found that the constitutively activated atfs-1 mutant et17 showed a marked decrease in food consumption, equivalent to that observed in nuo-6 and isp-1 mutants (Fig. 4F). Combined, this indicates that activation of ATFS-1 is sufficient to decrease food consumption, and is required for the diminished food intake in nuo-6 worms.

\section{The FOXO transcription factor DAF-16 is required for bacterial pathogen resistance in long-}

\section{lived mitochondrial mutants but does not account for activation of innate immunity genes}

As we have previously shown that target genes of the FOXO transcription factor DAF-16 are upregulated in the long-lived mitochondrial mutants(20), and others have shown that DAF-16 is required for the enhanced bacterial pathogen resistance of long-lived daf-2 mutants(38), we next examined the contribution of DAF-16 to bacterial pathogen resistance in long-lived 
mitochondrial mutants. We found that disruption of daf-16 completely abolished the increased resistance to bacterial pathogens in isp-1 worms (Fig. 5A), thereby indicating that DAF-16 is required for the enhanced bacterial pathogen resistance in isp-1 worms.

To determine the extent to which the effects of DAF-16 on bacterial pathogen resistance might be mediated through the same genes that are modulated by the p38-mediated innate immune signaling pathway, we examined the effect of daf-16 RNAi on the expression of innate immune genes in wild-type, nuo- 6 and isp-1 worms. While daf-16 RNAi was effective in decreasing the expression of DAF-16 target genes (sod-3, dod-3, mtl-1, ftn-1, icl-1, sodh-1) in all three strains, there was little or no effect on genes involved in innate immunity (T24B8.5, K08D8.5, F55G11.8, clec-65, clec-67, dod-22, Y9C9A.8 and C32H11.4) (Fig. 5B,C; Fig. S11). This finding is consistent with a previous study that compared microarray data of genes modulated by DAF-16 and PMK1 and observed no overlap, despite both pathways being required for pathogen resistance in daf-2 mutants(38). Thus, although DAF-16 activation contributes to the enhanced pathogen resistance in the long-lived mitochondrial mutants, it does not contribute to the upregulation of p38/ATF-7-regulated innate immunity genes in these worms.

\section{The mitoUPR transcription factor ATFS-1 mediates upregulation of innate immune genes in} long-lived mitochondrial mutants

In our previous studies, we have shown that the mitoUPR is activated in nuo-6 and isp-1 worms, and that this activation is required for their increased lifespan(19). As activation of the mitoUPR by RNAi against spg-7 (the worm homolog of SPG7/paraplegin) has been shown to increase resistance to PA14 and upregulate innate immunity genes(29), we hypothesized that the ATFS-1 activation that occurs in nuo-6 and isp-1 mutants(19) might contribute to their enhanced resistance to PA14.

To test this, we examined the effect of inhibiting the mitoUPR on the survival of nuo- 6 worms under bacterial pathogen stress. We found that disruption of atfs-1 completely ablates the 
bacterial pathogen resistance of nuo-6 worms such that nuo-6;atfs- 1 worms have decreased survival compared to wild-type or atfs-1 single mutants (Fig. 6A). This indicates that activation of the mitoUPR is required for the enhanced pathogen resistance in nuo-6 mutants.

To determine if ATFS-1 is also responsible for the upregulation of innate immunity genes in nuo6 mutants, we examined the effect of atfs-1 deletion. While deletion of atfs-1 did not decrease the expression of any of the innate immunity genes examined in wild-type worms, loss of atfs-1 markedly decreased the expression of innate immunity genes in nuo- 6 worms, in most cases reverting expression levels to wild-type (Fig. 6B). Thus, ATFS-1 is required for the upregulation of innate immunity genes in nuo-6 mutants, but is dispensable for the normal nutrient-driven regulation of innate immune gene expression that is seen in wild-type worms(39).

\section{Activation of ATFS-1 is sufficient to upregulate innate immune signaling genes}

Since loss of atfs-1 prevented the upregulation of innate immunity genes in nuo- 6 mutants, we next sought to determine if activation of ATFS-1 is sufficient to cause upregulation of innate immunity genes. To do this, we examined gene expression in two constitutively active atfs-1 mutants, et15 and et17(49). We found constitutive activation of ATFS-1 resulted in a significant upregulation of innate immunity genes (Fig. 7A). As a positive control, we found that the ATFS-1 target gene $h s p-6$ is significantly upregulated in both constitutively activated atfs-1 mutants (Fig. S12).

To determine if activation of ATFS-1 causes upregulation of innate immunity genes through activation of the p38-mediated innate immune signaling pathway, we examined PMK-1/p38 phosphorylation in a constitutively activated atfs-1 mutant (et17) and an atfs-1 loss-of-function mutant (gk3094). Similar to what we observed in the long-lived mitochondrial mutants, we found that the levels of PMK-1/p38 protein, phosphorylated PMK-1/p38 protein and the ratio of phosphorylated p38/p38 are equivalent to wild-type in both atfs-1 mutants (Fig. 7B). This 
393 suggests that activation of ATFS-1 can upregulate innate immunity genes without increased

394 activation of the p38-mediated innate immune signaling pathway.

396 To determine if ATFS-1 might be able to directly bind to the same innate immunity genes as

397 ATF-7, we compared data from two previous CHiP-seq studies(36, 50). For this comparison, we

398 defined innate immunity genes as genes that are upregulated in response to a 4-hour exposure

399 to PA14(36). After exposure to PA14, ATF-7 was found to be bound to 345 of these innate

400 immunity genes(36). Following exposure to mitochondrial stress induced by spg-7 RNAi, ATFS-1

401 was found to be bound to 51 of these innate immunity genes(50). Of the 51 innate immunity

402 genes that can be bound by ATFS-1, 49\% (25 genes) can also be bound by ATF-7 (Fig. S13; Table

403 S3). This binding likely occurs at different sites since the predicted binding site motifs of ATFS-1

404 and ATF-7 bear little similarity $(36,50)$. The fact that ATFS-1 and ATF-7 are able to bind to the

405 same genes involved in innate immunity indicates that the mitoUPR and p38-mediated innate

406 immune signaling pathway act in parallel to modulate the expression of innate immunity genes,

407 which are critical for lifespan extension. 


\section{Discussion}

Aging may be defined as a progressive decline of physiologic functions that increases the

probability of death. The major factors that contribute to aging have been categorized into

412 hallmarks or pillars of aging(51). Interestingly, at least two of the seven pillars are directly

413 related to the immune system, but in opposite directions. First, the ability of the immune

414 system to fight off external stresses such as exposure to bacterial pathogens declines with age,

415 which can be accounted for by an age-dependent decrease in basal and activated PMK-1/p38

416 levels $(52,53)$. Second, there is an increase in the basal activity of the immune system that

417 occurs with advancing age, eventually leading to chronic inflammation(54). Thus, the

418 relationship between aging and the immune response can be seen as a fine balance between

419 maintaining the ability to respond to stresses, while limiting non-specific immune activation.

421 Our ability to precisely define the relationship between immune activity and aging has been greatly enhanced by the discovery of genes that increase lifespan in model organisms, which

423 allow for investigation of the molecular mechanisms. In this work, we demonstrate that mild impairment of mitochondrial function through mutations affecting either complex I (nuo-6) or

425 complex III (isp-1) of the electron transport chain results in increased expression of a broad panel of genes involved in innate immunity. Treatment with the complex I inhibitor rotenone

427 was previously shown to cause neurodegeneration and the upregulation of a small panel of genes involved in innate immunity, but the mechanism by which these genes were upregulated was not determined(37). While it is well established that the degeneration of neurons can activate an innate immune response $(55,56)$, our work clearly demonstrates that the

431 impairment of mitochondrial function can also lead to the upregulation of innate immunity

432 genes. Further, our results indicate that the upregulation of innate immunity genes in response

433 to mild mitochondrial impairment is dependent on the mitoUPR transcription factor ATFS-1 and 434 all of the components of the p38-mediated innate immune signaling pathway (NSY-1, SEK-1, 435 PMK-1, ATF-7). 
437 While both the long-lived mitochondrial mutants and constitutively active atfs-1 mutants, exhibit increased expression of ATF-7 target genes overall, these mutants show no increase in p38-mediated innate immune signaling (Fig. 7). Moreover, since ATFS-1 activation is sufficient to decrease food intake, these mutants would be predicted to have decreased ATF-7 target gene expression because decreasing food intake diminishes p38-mediated innate immune

442 signaling(39). Combined this suggests that ATFS-1 can activate innate immunity genes directly.

443 This conclusion is supported by the fact that ATFS-1 can bind to the same innate immunity genes as ATF-7 (Fig. S12).

Previous work examining the relationship between the mitoUPR and p38-mediated innate 447 immune signaling pathway has generated conflicting results. In an earlier study, activation of the mitoUPR by spg-7 RNAi was found to increase the survival of pmk-1 and sek-1 mutants on PA14, leading the authors to conclude that the mitoUPR was increasing pathogen resistance independently of the p38-mediated innate immune signaling pathway(29). Subsequently, it was shown that overexpression of the mitoUPR target gene $h s p-60$ is sufficient to activate the p38mediated innate immune signaling pathway leading to increased expression of genes involved

453 in innate immunity and increased PA14 resistance, all of which was dependent on p38-

454 mediated innate immune signaling pathway (nsy-1, sek-1 and pmk-1)(40). Our current results resolve this discrepancy by demonstrating that the mitoUPR can affect the expression of innate

456 immunity genes and pathogen resistance both directly through binding of ATFS-1 to innate

457 immunity genes, and indirectly through the p38-mediated innate immune signaling and ATF-7

458 (Fig. 7).

The increased expression of innate immunity genes in nuo- 6 and isp-1 worms results in enhanced resistance to bacterial pathogens, which is dependent on both ATFS-1 and the p38-

462 mediated innate immune signaling pathway. The increased resistance to bacterial pathogens is 463 also dependent on DAF-16, which has increased nuclear localization in long-lived mitochondrial 464 mutants(20). However, the mechanism by which DAF-16 protects against bacterial pathogens appears to be distinct from the mechanism of ATFS-1, as loss of DAF-16 does not affect the 
expression of the innate immunity genes that are upregulated by ATFS-1 and ATF-7 (Fig. 5; Fig. S10)(38). The effect of DAF-16 on bacterial pathogen resistance may instead be due to a general effect of DAF-16 on resistance to stress.

We have previously shown that both ATFS-1 and DAF-16 are required for the long-lifespan of nuo-6 and isp-1 mutants $(19,20)$. Here, we show that their lifespan is also completely

472 dependent on the p38-mediated innate immune signaling pathway (NSY-1, SEK-1, PMK-1, ATF-

473 7). As all of these factors are also required for the enhanced bacterial pathogen resistance in

474 nuo-6 and isp-1 worms, this indicates that there are multiple conserved genetic pathways

475 acting in parallel to upregulate innate immunity and promote longevity in response to impaired

476 mitochondrial function.

478 It appears that there are multiple ways to optimize innate immune signaling with respect to 479 longevity. As in the long-lived mitochondrial mutants that we studied here, the p38-mediated 480 innate immune signaling pathway is also required for the longevity of other genes and 481 interventions that increase lifespan. daf-2 mutants have increased lifespan(1) and increased resistance to bacterial pathogens(57). Unlike the nuo- 6 and isp-1 mutants, daf-2 mutants show a downregulation of many, but not all, SEK-1-dependent genes involved in innate immunity through decreased feeding and decreased phosphorylation of PMK-1/p38(39). Nonetheless, 485 genes involved in the p38-mediated innate immune signaling pathway (SEK-1, PMK-1) are required for the long-lifespan, and increased resistance to bacterial pathogens in daf-2 worms,

487 as is DAF-16(28, 38, 58).

Similar to daf-2 mutants, the increase in lifespan caused by dietary restriction resulting from decreased nutrient availability is dependent on the p38-mediated innate immune signaling pathway (NSY-1, SEK-1, PMK-1, ATFS-7), despite the fact that dietary restriction was found to decrease PMK-1/p38 activation and downregulate more innate immune genes (162 genes) than were upregulated (46 genes)(39). Based on this and other supporting data it was concluded that lifespan extension resulting from dietary restriction was not mediated by pathogen 
495 resistance per se, but by a metabolic/nutrient-related function of the p38-mediated innate

496 immune signaling pathway.

497

498 Combined, this clearly indicates that it is not a simple relationship between innate immune

499 activation and maximum longevity, as increased lifespan can be achieved with upregulation or

500 downregulation of innate immunity genes. While a functional p38-mediated innate immune

501 signaling pathway may be universally required for long life, different levels of its activity are

502 required to achieve maximum lifespan extension under different circumstances or genetic

503 backgrounds. While many previous studies have only examined a few genes involved in innate

504 immunity, the fact that different innate immune genes can be both upregulated and

505 downregulated in the same mutants demonstrates the importance of looking more broadly

506 across all genes involved in innate immunity to gain a more in-depth understanding of how

507 these changes are affecting longevity. Moreover, it will be important to understand the specific

508 roles of each of these genes in pathogen defense and longevity. 


\section{Conclusions}

511 Overall, this work demonstrates the importance of the p38-mediated innate immune signaling

512 pathway and the mitochondrial unfolded protein response for both pathogen resistance and

513 lifespan. It shows that activation of innate immunity is a key function of the mitoUPR that works

514 in concert with the p38-mediated innate immune signaling pathway as a key defense

515 mechanism, which is activated by mitochondrial impairment. The relationship between innate

516 immune gene expression, pathogen resistance and lifespan is complex, and there are multiple

517 ways to optimize the levels of innate immune activation with respect to longevity. As all of the

518 signaling pathways studied here are conserved up to mammals, advancing our understanding of

519 the links between innate immune gene expression, pathogen resistance and longevity in model

520 organisms, may advance our understanding of these processes in humans. Understanding the

521 relationship between mitochondrial function, innate immunity and longevity will provide

522 important insights into fundamental aspects of how lifespan can be extended. 


\section{Materials and Methods}

524

525 Strains

526 WT(N2)

527 MQ1333 nuo-6(qm200)

528 JVR171 isp-1(qm150)

529 T24B8.5p::GFP

$530 \quad$ JVR521 nsy-1(ok593)

531 JVR520 sek-1(km4)

532 JVR165 pmk-1(km25)

533 ZD442 atf-7(qd22); agls219[T24B8.5p::GFP+ttx-3p::GFP]

534 JVR455 atfs-1(gk3094)

535 JVR479 nuo-6(qm200);atfs-1(gk3094)

536 QC1115 atfs-1(et15)

537 QC1117 atfs-1(et17)

538 JVR533 nuo-6(qm200);nsy-1(ok593)

539 JVR543 nuo-6(qm200);sek-1(km4)

540 JVR281 nuo-6(qm200);pmk-1(km25)

541 JVR527 nuo-6(qm200);atf-7(qd22); agls219[T24B8.5p::GFP+ttx-3p::GFP]

542 JVR532 isp-1(qm150);nsy-1(ok593)

543 JVR534 isp-1(qm150);sek-1(km4)

544 JVR538 isp-1(qm150);atf-7(qd22); agls219[T24B8.5p::GFP+ttx-3p::GFP]

545 math-33(tm6724)

546 JVR456 isp-1(qm150); math-33(tm6724)

547 JVR457 nuo-6(qm200); math-33(tm6724)

548 daf-16(mu86)

549 JVR380 isp-1(qm150);daf-16(mu86) 
551 Strains were grown on OP50 bacteria at $20^{\circ} \mathrm{C}$. All of the strains were genotyped or sequenced

552 to confirm the presence of the mutation in the gene of interest. Double mutants were

553 generated by crossing WT males to nuo- 6 or isp-1 mutants and crossing the resulting

554 heterozygous male progeny (nuo-6/+ or isp-1/+) to the mutant of interest (nsy-1, sek-1, pmk-1,

555 atf-7). After selfing, slow growing progeny were singled and genotyped or sequenced to identify

556 double mutants. We were unable to generate isp-1 pmk-1 and nuo-6 daf-16 double mutants

557 due to the close proximity of these two genes on the same chromosome.

558

Bacteria pathogenesis - slow kill assay

560 Pathogenesis assays with $P$. aeruginosa strain PA14 were performed as described previously(39). In brief, the overnight PA14 culture was seeded to the center of a 35-mm NGM agar plate containing $20 \mathrm{mg} / \mathrm{L}$ FUdR. Seeded plates were incubated at $37^{\circ} \mathrm{C}$ overnight, then incubated at room temperature overnight. Approximately 40 day three adults were transferred to these prepared plates, with 10 plates scored per strain. The assays were conducted at $20^{\circ} \mathrm{C}$. Animals that did not respond to gentle prodding from a platinum wire were scored as dead.

\section{Lifespan}

568

All lifespan assays were performed at $20^{\circ} \mathrm{C}$. Except where noted lifespan assays were performed on NGM plates seeded with OP50 bacteria. Lifespan assays included FUdR to limit the development of progeny. Lifespan assays on solid plates utilized $25 \mu \mathrm{M}$ FUdR to minimize

571 potential effects of FUdR on lifespan(59). Animals were excluded from the experiment if they

572 crawled off the plate or died of internal hatching of progeny or expulsion of internal organs.

573 Lifespan assays with arrested bacteria. Growth-arrested bacterial food was prepared through

574 treatment with antibiotics and cold as described previously(39). All worms were passaged at

$57520^{\circ} \mathrm{C}$ for at least two generations before lifespan assays were initiated. Synchronized

576 populations of L1 animals were obtained by hypochlorite treatment, then allowed to develop at

$57720^{\circ} \mathrm{C}$ on standard NGM plates seeded with OP50-1. The young adults were transferred to NGM

578 agar plates containing $50 \mathrm{mg} / \mathrm{L}$ ampicillin, $10 \mathrm{mg} / \mathrm{L}$ kanamycin, $1 \mathrm{mg} / \mathrm{L}$ tetracycline, $50 \mathrm{mg} / \mathrm{L}$

579 nystatin, and $100 \mathrm{mg} / \mathrm{L} \mathrm{FUdR}$, and seeded with the cold- and antibiotic- treated OP50-1. Three 
days later the animals were washed twice in S basal, and transferred to a 24 well plate with 1 $\mathrm{mL} \mathrm{S}$ basal in each well containing the arrested OP50-1 at $\mathrm{OD}_{600} 3.20-40$ worms were placed in each well.

\section{Western Blotting}

Protein levels were measured by western blotting in whole worm lysates obtained from four independent biological replicates. Briefly, whole worm lysates obtained from approximately 1000 animals on day 1 of adulthood were subjected to SDS-PAGE in polyacrylamide gels (4\%12\%), immunoblotted with specific antibodies against phospho-p38 (Cell Signaling) or total p38/PMK-1(47), and visualized following standard procedures. Quantification analysis of all blots was performed with the use of ImageJ software. Results were corrected to Ponceau red staining $(0.5 \%, \mathrm{w}: \mathrm{v})$ of the membrane since nuo- 6 worms were found to exhibit decreased levels of tubulin protein.

\section{Food intake assay}

Food intake was quantified in liquid culture by measuring the relative amount of growtharrested bacteria that are present in a culture before and after incubation with $C$. elegans, as previously described(39). Synchronized populations of L1 animals were obtained by hypochlorite treatment and allowed to develop at $20^{\circ} \mathrm{C}$ on standard NGM agar plates seeded with OP50. The worms were transferred onto NGM plates containing FUdR at the L4 stage of development. On day 3 of adulthood, animals were washed twice in S basal $(5.85 \mathrm{~g} / \mathrm{L} \mathrm{NaCl}, 1$ g/L K2HPO4, 6 g/L KH2PO4) and 30-50 worms were transferred to each well of a 24 well plate containing $1 \mathrm{~mL}$ of arrested OP50 at optical density $3(600 \mathrm{~nm})$ re-suspended in S basal complete medium (S basal containing $5 \mathrm{mg} / \mathrm{L}$ cholesterol, $50 \mathrm{mg} / \mathrm{L}$ ampicillin, $10 \mathrm{mg} / \mathrm{L}$ kanamycin, $1 \mathrm{mg} / \mathrm{L}$ tetracycline, $50 \mathrm{mg} / \mathrm{L}$ nystatin, FUdR). On day 12 of adulthood, bacteria were removed from the wells by washing and diluted 10x with $\mathrm{S}$ basal prior to measuring the optical density $(600 \mathrm{~nm})$. The relative food intake was determined by the change in OP50 optical density between day 3 and day 12 of adulthood, and normalized to the number of worms per well. 


\section{Bacterial avoidance assay}

611 A colony of PA14 was placed in $5 \mathrm{ml}$ of Luria Broth (LB) or a colony of OP50 was placed in $5 \mathrm{ml}$

$6122 \mathrm{YT}$, and the cultures were incubated, shaking, for $16 \mathrm{hr}$ at $37^{\circ} \mathrm{C} .100 \mathrm{ul}$ of the culture was

613 seeded onto the center of $6 \mathrm{~cm}$ NGM plates, which were grown for 24 hours at room

614 temperature. Animals were synchronized by picking stage $L 4$ animals the day before the experiment and were placed on OP50 plates at $20^{\circ} \mathrm{C}$. The synchronized day 1 animals were

616 washed off of the OP50 plates with M9 buffer, washed three times in M9 buffer, and then $~ 40$

617 animals were deposited on each of the assay plates using a glass pipette. The animals were 618 placed on the agar $1 \mathrm{~cm}$ from the edge of the bacterial spots. The plates were then placed at

$61920^{\circ} \mathrm{C}$. The number of animals on and off of the bacteria were counted after 24 hours.

\section{Quantification of reporter fluorescence}

622 T24B8.5p::GFP animals were randomly selected and imaged with ZEN 2012 software on an Axio 623 Imager M2 microscope with a 10X/0.25 objective (Zeiss, Jena, Germany). Fluorescence 624 brightness was quantified blindly using NIH ImageJ software.

\section{RNA isolation}

627 mRNA was collected from pre-fertile young adult worms using Trizol as previously described

628 (60). For quantitative real-time RT-PCR, we collected three biological replicates for each strain on separate days. For RNA sequencing experiments, we collected 3-6 biological replicates.

\section{Quantitative Real-Time RT-PCR}

632 The mRNA was converted to cDNA using a High-Capacity cDNA Reverse Transcription kit (Life 633 Technologies/Invitrogen) according to the manufacturer's directions. qPCR was performed 634 using a FastStart Universal SYBR Green kit (Roche) in a Bio-Rad iCycler real-time PCR detection 635 system (61). Primer sequences for other genes tested include:

636 T24B8.5 (TACACTGCTTCAGAGTCGTG, CGACAACCACTTCTAACATCTG); 
K08D8.5 (CAAAATATCCTCCGGGAAGTC, TTCACGGAATCACCATCGTA);

F55G11.8 (GGAAATGGTTGCAAACTTGG, TGCAGAATCGACAGTTTGGA);

clec-67 (TTTGGCAGTCTACGCTCGTT, CTCCTGGTGTGTCCCATTTT);

640

clec-65 (GCAATCAACCTCGTGATGTG, CGCAGAAGCAGTTTGTATCC);

dod-22 (TCCAGGATACAGAATACGTACAAGA, GCCGTTGATAGTTTCGGTGT);

\section{RNA sequencing and Bioinformatic Analysis}

RNA sequencing was performed as previously described(62). RNA-seq data is available on NCBI GEO: GSE93724(20), GSE110984(19) and was analyzed by the Harvard School of Public Health Bioinformatics core for this paper.

649 Read mapping and expression level estimation. All samples were processed using an RNA-seq

650 pipeline implemented in the bcbio-nextgen project (https://bcbio-

nextgen.readthedocs.org/en/latest/). Raw reads were examined for quality issues using FastQC

652 (http://www.bioinformatics.babraham.ac.uk/projects/fastqc/) to ensure library generation and sequencing data were suitable for further analysis. If necessary, adapter sequences, other contaminant sequences such as polyA tails and low quality sequences were trimmed from reads using cutadapt http://code.google.com/p/cutadapt/. Trimmed reads were aligned to the Ensembl build WBcel235 (release 90) of the C.elegans genome using STAR(63). Quality of alignments was assessed by checking for evenness of coverage, rRNA content, genomic context of alignments (for example, alignments in known transcripts and introns), complexity and other quality checks. Expression quantification was performed with Salmon(64) to identify transcript-

660 level abundance estimates and then collapsed down to the gene-level using the R Bioconductor package tximport(65). Principal components analysis (PCA) and hierarchical clustering methods

662 validated clustering of samples from the same batches and across different mutants.

664 performed at the gene level using the R Bioconductor package DESeq2(66). For each wildtype- 
datasets in which experiments were run across two batches, we included batch as a covariate in

667 the linear model.

668 Overlap table. Lists of differentially expressed genes were separated by direction of expression 669 change and compared to genes that are modulated in response to exposure to the bacterial 670 pathogen $P$. aeruginosa strain PA14 in a PMK-1 and ATF-7-dependent manner, as identified by

671 Fletcher et al., 2019(36). Significance of overlap was computed using the hypergeometric test.

672 Heatmaps. For each dataset (long-lived mutant comparison to wildtype), the raw counts were

673 regularized log (rlog) transformed using the DESeq2 package(66). This transformation

674 moderates the variance across the mean, improving the clustering in an unbiased manner. The

675 rlog matrix was then subset to retain only those genes from the Fletcher et al gene lists which

676 were also significantly differentially expressed between mutant and wildtype. Heatmaps were

677 plotted using the pheatmap package in $\mathrm{R}$.

678

\section{Statistical Analysis}

680 Experiments were conducted such that the experimenter was blinded to the genotype of the

681 animals being tested. The worms for each experiment were randomly selected from

682 maintenance plates at stages when all worms appeared healthy. Except where noted, we

683 completed a minimum of three biological replicates for each assay (independent population of worms tested on a different day). Statistically significance of differences between groups of

685 more than two was determined by one-way ANOVA with Dunnett's multiple comparison post-

686 hoc test or two-way ANOVA with Bonferroni post-doc test using Graphpad Prism. For survival

687 analysis, a log-rank test was used. On all bar graphs, the bar indicates the average (mean) and

688 error bars indicate standard error of the mean (SEM). This study was not pre-registered. No

689 sample size calculations were performed. This study did not include a pre-specified primary 690 endpoint. 
702

703

704

705

706

707

708

709

710

711

712

713

714

715

716

717

718

719

Acknowledgments. Some strains were provided by the CGC, which is funded by NIH Office of Research Infrastructure Programs (P30 OD010440). We would also like to acknowledge the $C$. elegans knockout consortium and the National Bioresource Project of Japan for providing strains used in this research.

Competing interests. The authors have declared that no competing interests exist.

Author Contributions. Conceptualization: JVR, TKB. Methodology: JCC, ZW, PR, SS, MM, TKB, JVR. Investigation: JCC, ZW, PR, SS, MM, JVR. Visualization: JCC, ZW, PR, SS, MM, JVR. Writing original draft: JVR. Writing - review and editing: JCC, ZW, PR, SS, MM, TKB, JVR. Supervision: JCBF, TKB, JVR.

Materials \& Correspondence. Correspondence and material requests should be addressed to Jeremy Van Raamsdonk.

Data availability. RNA-seq data has been deposited on GEO: GSE93724, GSE110984. All other data and strains generated in the current study are included with the manuscript or available from the corresponding author on request.

Funding. This work was supported by the National Institute of General Medical Sciences (NIGMS; https://www.nigms.nih.gov/; JVR) by grant number R01 GM121756, the Canadian Institutes of Health Research (CIHR; http://www.cihr-irsc.gc.ca/ ; JVR) and the Natural Sciences and Engineering Research Council of Canada (NSERC; https://www.nserccrsng.gC.ca/index eng.asp; JVR). TKB, JCC, and ZW were supported by research (R35 GM122610, R01 AG054215) and center (P30 DK036836) grants from the NIH. JCC was supported by Fundação de Amparo à Pesquisa do Estado de São Paulo (FAPESP 2019/18444-9). The funders had no role in study design, data collection and analysis, decision to publish, or preparation of the manuscript. 
750

751

752

753

754

755

756

757

758

759

760

761

762

763

764

765

766

\section{References}

1. C. Kenyon, J. Chang, E. Gensch, A. Rudner, R. Tabtiang, A C. elegans mutant that lives twice as long as wild type. Nature 366, 461-464 (1993).

2. M. R. Klass, Aging in the nematode Caenorhabditis elegans: major biological and environmental factors influencing life span. Mech Ageing Dev 6, 413-429 (1977).

3. D. B. Friedman, T. E. Johnson, A mutation in the age-1 gene in Caenorhabditis elegans lengthens life and reduces hermaphrodite fertility. Genetics 118, 75-86 (1988).

4. D. J. Clancy et al., Extension of life-span by loss of CHICO, a Drosophila insulin receptor substrate protein. Science 292, 104-106 (2001).

5. M. Holzenberger et al., IGF-1 receptor regulates lifespan and resistance to oxidative stress in mice. Nature 421, 182-187 (2003).

6. F. Flachsbart et al., Identification and characterization of two functional variants in the human longevity gene FOXO3. Nature communications 8, 2063 (2017).

7. A. Wong, P. Boutis, S. Hekimi, Mutations in the clk-1 gene of Caenorhabditis elegans affect developmental and behavioral timing. Genetics 139, 1247-1259 (1995).

8. B. Lakowski, S. Hekimi, Determination of life-span in Caenorhabditis elegans by four clock genes. Science 272, 1010-1013 (1996).

9. J. Feng, F. Bussiere, S. Hekimi, Mitochondrial electron transport is a key determinant of life span in Caenorhabditis elegans. Developmental cell 1, 633-644 (2001).

10. W. Yang, S. Hekimi, Two modes of mitochondrial dysfunction lead independently to lifespan extension in Caenorhabditis elegans. Aging cell 9, 433-447 (2010).

11. A. Dillin et al., Rates of behavior and aging specified by mitochondrial function during development. Science 298, 2398-2401 (2002).

12. S. S. Lee et al., A systematic RNAi screen identifies a critical role for mitochondria in C. elegans longevity. Nature genetics 33, 40-48 (2003).

13. J. M. Copeland et al., Extension of Drosophila life span by RNAi of the mitochondrial respiratory chain. Curr Biol 19, 1591-1598 (2009).

14. X. Liu et al., Evolutionary conservation of the clk-1-dependent mechanism of longevity: loss of mclk1 increases cellular fitness and lifespan in mice. Genes \& development 19, 2424-2434 (2005).

15. C. Dell'agnello et al., Increased longevity and refractoriness to $\mathrm{Ca}(2+)$-dependent neurodegeneration in Surf1 knockout mice. Hum Mol Genet 16, 431-444 (2007).

16. W. Yang, S. Hekimi, A mitochondrial superoxide signal triggers increased longevity in Caenorhabditis elegans. PLoS biology 8, e1000556 (2010).

17. J. M. Van Raamsdonk, S. Hekimi, Superoxide dismutase is dispensable for normal animal lifespan. Proc Natl Acad Sci U S A 109, 5785-5790 (2012).

18. C. Yee, W. Yang, S. Hekimi, The Intrinsic Apoptosis Pathway Mediates the Pro-Longevity Response to Mitochondrial ROS in C. elegans. Cell 157, 897-909 (2014).

19. Z. Wu et al., Mitochondrial unfolded protein response transcription factor ATFS-1 promotes longevity in a long-lived mitochondrial mutant through activation of stress response pathways. BMC biology 16, 147 (2018).

20. M. M. Senchuk et al., Activation of DAF-16/FOXO by reactive oxygen species contributes to longevity in long-lived mitochondrial mutants in Caenorhabditis elegans. PLoS Genet 14, e1007268 (2018).

21. E. Munkacsy et al., DLK-1, SEK-3 and PMK-3 Are Required for the Life Extension Induced by Mitochondrial Bioenergetic Disruption in C. elegans. PLoS Genet 12, e1006133 (2016). 
22. L. Walter, A. Baruah, H. W. Chang, H. M. Pace, S. S. Lee, The homeobox protein CEH-23 mediates prolonged longevity in response to impaired mitochondrial electron transport chain in C. elegans. PLoS biology 9, e1001084 (2011).

23. A. Baruah et al., CEP-1, the Caenorhabditis elegans p53 Homolog, Mediates Opposing Longevity Outcomes in Mitochondrial Electron Transport Chain Mutants. PLoS Genet 10, e1004097 (2014).

24. R. G. Tepper et al., PQM-1 Complements DAF-16 as a Key Transcriptional Regulator of DAF-2Mediated Development and Longevity. Cell 154, 676-690 (2013).

25. C. T. Murphy et al., Genes that act downstream of DAF-16 to influence the lifespan of Caenorhabditis elegans. Nature 424, 277-283 (2003).

26. V. Jovaisaite, L. Mouchiroud, J. Auwerx, The mitochondrial unfolded protein response, a conserved stress response pathway with implications in health and disease. The Journal of experimental biology 217, 137-143 (2014).

27. A. M. Nargund, M. W. Pellegrino, C. J. Fiorese, B. M. Baker, C. M. Haynes, Mitochondrial import efficiency of ATFS-1 regulates mitochondrial UPR activation. Science 337, 587-590 (2012).

28. D. J. Dues, E. K. Andrews, M. M. Senchuk, J. M. Van Raamsdonk, Resistance to Stress Can Be Experimentally Dissociated From Longevity. The journals of gerontology. Series A, Biological sciences and medical sciences 74, 1206-1214 (2019).

29. M. W. Pellegrino et al., Mitochondrial UPR-regulated innate immunity provides resistance to pathogen infection. Nature 516, 414-417 (2014).

30. J. A. Hoffmann, F. C. Kafatos, C. A. Janeway, R. A. Ezekowitz, Phylogenetic perspectives in innate immunity. Science 284, 1313-1318 (1999).

31. D. A. Kimbrell, B. Beutler, The evolution and genetics of innate immunity. Nature reviews. Genetics 2, 256-267 (2001).

32. J. E. Irazoqui, J. M. Urbach, F. M. Ausubel, Evolution of host innate defence: insights from Caenorhabditis elegans and primitive invertebrates. Nat Rev Immunol 10, 47-58 (2010).

33. D. H. Kim et al., A conserved p38 MAP kinase pathway in Caenorhabditis elegans innate immunity. Science 297, 623-626 (2002).

34. D. H. Kim, J. J. Ewbank, Signaling in the innate immune response. WormBook 2018, 1-35 (2018).

35. R. P. Shivers et al., Phosphorylation of the conserved transcription factor ATF-7 by PMK-1 p38 MAPK regulates innate immunity in Caenorhabditis elegans. PLoS Genet 6, e1000892 (2010).

36. M. Fletcher, E. J. Tillman, V. L. Butty, S. S. Levine, D. H. Kim, Global transcriptional regulation of innate immunity by ATF-7 in C. elegans. PLoS Genet 15, e1007830 (2019).

37. M. R. Chikka, C. Anbalagan, K. Dvorak, K. Dombeck, V. Prahlad, The Mitochondria-Regulated Immune Pathway Activated in the C. elegans Intestine Is Neuroprotective. Cell reports 16, 23992414 (2016).

38. E. R. Troemel et al., p38 MAPK regulates expression of immune response genes and contributes to longevity in C. elegans. PLoS Genet 2, e183 (2006).

39. Z. Wu et al., Dietary Restriction Extends Lifespan through Metabolic Regulation of Innate Immunity. Cell Metab 29, 1192-1205 e1198 (2019).

40. D. E. Jeong et al., Mitochondrial chaperone HSP-60 regulates anti-bacterial immunity via p38 MAP kinase signaling. EMBO J 36, 1046-1065 (2017).

41. D. H. Block et al., The Developmental Intestinal Regulator ELT-2 Controls p38-Dependent Immune Responses in Adult C. elegans. PLoS Genet 11, e1005265 (2015).

42. M. W. Tan, L. G. Rahme, J. A. Sternberg, R. G. Tompkins, F. M. Ausubel, Pseudomonas aeruginosa killing of Caenorhabditis elegans used to identify $P$. aeruginosa virulence factors. Proc Natl Acad Sci U S A 96, 2408-2413 (1999). 
43. M. W. Tan, S. Mahajan-Miklos, F. M. Ausubel, Killing of Caenorhabditis elegans by Pseudomonas aeruginosa used to model mammalian bacterial pathogenesis. Proc Natl Acad Sci U S A 96, 715720 (1999).

44. N. V. Kirienko, B. O. Cezairliyan, F. M. Ausubel, J. R. Powell, Pseudomonas aeruginosa PA14 pathogenesis in Caenorhabditis elegans. Methods Mol Biol 1149, 653-669 (2014).

45. D. Garigan et al., Genetic analysis of tissue aging in Caenorhabditis elegans: a role for heat-shock factor and bacterial proliferation. Genetics 161, 1101-1112 (2002).

46. D. Gems, D. L. Riddle, Genetic, behavioral and environmental determinants of male longevity in Caenorhabditis elegans. Genetics 154, 1597-1610 (2000).

47. H. Inoue et al., The C. elegans p38 MAPK pathway regulates nuclear localization of the transcription factor SKN-1 in oxidative stress response. Genes \& development 19, 2278-2283 (2005).

48. J. M. Hourihan, L. E. Moronetti Mazzeo, L. P. Fernandez-Cardenas, T. K. Blackwell, Cysteine Sulfenylation Directs IRE-1 to Activate the SKN-1/Nrf2 Antioxidant Response. Molecular cell 63, 553-566 (2016).

49. M. Rauthan, P. Ranji, N. Aguilera Pradenas, C. Pitot, M. Pilon, The mitochondrial unfolded protein response activator ATFS-1 protects cells from inhibition of the mevalonate pathway. Proc Natl Acad Sci U S A 110, 5981-5986 (2013).

50. A. M. Nargund, C. J. Fiorese, M. W. Pellegrino, P. Deng, C. M. Haynes, Mitochondrial and nuclear accumulation of the transcription factor ATFS-1 promotes OXPHOS recovery during the UPR $(\mathrm{mt})$. Mol Cell 58, 123-133 (2015).

51. B. K. Kennedy et al., Geroscience: linking aging to chronic disease. Cell 159, 709-713 (2014).

52. D. J. Dues et al., Aging causes decreased resistance to multiple stresses and a failure to activate specific stress response pathways. Aging (Albany NY) 8, 777-795 (2016).

53. M. J. Youngman, Z. N. Rogers, D. H. Kim, A decline in p38 MAPK signaling underlies immunosenescence in Caenorhabditis elegans. PLoS Genet 7, e1002082 (2011).

54. C. Franceschi et al., Inflammaging and anti-inflammaging: a systemic perspective on aging and longevity emerged from studies in humans. Mech Ageing Dev 128, 92-105 (2007).

55. L. I. Labzin, M. T. Heneka, E. Latz, Innate Immunity and Neurodegeneration. Annu Rev Med 69, 437-449 (2018).

56. M. T. Heneka, M. P. Kummer, E. Latz, Innate immune activation in neurodegenerative disease. Nat Rev Immunol 14, 463-477 (2014).

57. D. A. Garsin et al., Long-lived C. elegans daf-2 mutants are resistant to bacterial pathogens. Science 300, 1921 (2003).

58. K. Zarse et al., Impaired insulin/IGF1 signaling extends life span by promoting mitochondrial Lproline catabolism to induce a transient ROS signal. Cell metabolism 15, 451-465 (2012).

59. J. M. Van Raamsdonk, S. Hekimi, FUdR causes a twofold increase in the lifespan of the mitochondrial mutant gas-1. Mech Ageing Dev 132, 519-521 (2011).

60. C. E. Schaar et al., Mitochondrial and cytoplasmic ROS have opposing effects on lifespan. PLoS Genet 11, e1004972 (2015).

61. E. Machiela, D. J. Dues, M. M. Senchuk, J. M. Van Raamsdonk, Oxidative stress is increased in C. elegans models of Huntington's disease but does not contribute to polyglutamine toxicity phenotypes. Neurobiology of disease 96, 1-11 (2016).

62. D. J. Dues et al., Uncoupling of oxidative stress resistance and lifespan in long-lived isp-1 mitochondrial mutants in Caenorhabditis elegans. Free radical biology \& medicine 108, 362-373 (2017).

63. A. Dobin et al., STAR: ultrafast universal RNA-seq aligner. Bioinformatics 29, 15-21 (2013). 
bioRxiv preprint doi: https://doi.org/10.1101/2021.03.31.437812; this version posted April 1, 2021. The copyright holder for this preprint (which

was not certified by peer review) is the author/funder, who has granted bioRxiv a license to display the preprint in perpetuity. It is made available under aCC-BY 4.0 International license.

860 64. R. Patro, G. Duggal, M. I. Love, R. A. Irizarry, C. Kingsford, Salmon provides fast and bias-aware quantification of transcript expression. Nat Methods 14, 417-419 (2017).

863

65. C. Soneson, M. I. Love, M. D. Robinson, Differential analyses for RNA-seq: transcript-level estimates improve gene-level inferences. F1000Res 4, 1521 (2015).

865

66. M. I. Love, W. Huber, S. Anders, Moderated estimation of fold change and dispersion for RNAseq data with DESeq2. Genome Biol 15, 550 (2014).

866 


\section{Figure Legends}

Figure 1. Innate immunity genes are upregulated in long-lived mitochondrial mutants. A.

Genes involved in innate immunity are significantly upregulated in nuo-6 and isp-1 worms.

871 Gene expression changes in the mitochondrial mutants were determined by RNA sequencing of

872 six biological replicates and compared to wild-type N2 worms. Results represent counts per

873 million (CPM) expressed as a percentage of wild-type. B. Upregulation of innate immunity

874 genes is dependent on the p38-mediated innate immune signaling pathway including NSY-1,

875 SEK-1, PMK-1 and ATF-7. Gene expression changes were examined by quantitative RT-PCR with

8763 biological replicates per strain. C. Using a fluorescent reporter strain for the innate immunity

877 gene T24B8.5 confirms that innate immunity genes are upregulated in the long-lived

878 mitochondrial mutants and that components of the p38-mediated innate immune signaling

879 pathway are required for their upregulation. D. Differentially expressed genes in long-lived

880 mitochondrial mutants show significant overlap with genetic targets of the p38-mediated

881 innate immune signaling pathway. Significantly modulated genes in long-lived mitochondrial

882 mutants nuo-6 and isp-1 (six biological replicate per strain) were compared to genes that are

883 modulated in response to exposure to the bacterial pathogen $P$. aeruginosa strain PA14 in a

884 PMK-1- and ATF-7-dependent manner as identified by Fletcher et al., 2019. There is a highly

885 significantly degree of overlap between genes upregulated by activation of the p38-mediated

886 innate immune pathway and genes upregulated in nuo-6 and isp-1 mutants. Similarly, there is a

887 highly significantly degree of overlap between genes downregulated by activation of the p38-

888 mediated innate immune pathway and genes downregulated in nuo- 6 and isp-1 mutants. Error bars indicate SEM. ${ }^{*} p<0.05, * * p<0.01, * * * p<0.001$. 


\section{Figure 2. Long-lived mitochondrial mutants exhibit increased resistance to bacterial}

893 pathogens that is dependent on presence of p38-mediated innate immune signaling pathway.

894 Resistance to bacterial pathogens was tested by exposing worms to Pseudomonas aeruginosa

895 strain PA14 in a slow kill assay. A. Both nuo-6 and isp-1 long-lived mitochondrial mutants

896 showed increased resistance compared to wild-type worms. Disruption of components of the

897 p38-mediated innate immune signaling pathway (nsy-1, sek-1, pmk-1, atf-7) markedly

898 decreases resistance to PA14 in wild-type (B), nuo-6 (C) and isp-1 (D) worms. Statistical analysis

899 on survival plots was performed with log-rank test. Significance is indicated between the strain

900 listed on top and all other strains. $* * * p<0.001$. All strains were tested in a single parallel

901 experiment. Data from panel A is repeated in panels B, C, and D for direct comparison. Raw

902 data and $\mathbf{N}$ for each strain can be found in Table S2.

903

904 
905 Figure 3. Disruption of the p38-mediated innate immune signaling pathway markedly ablates

906 extended longevity of long-lived mitochondrial mutants. To examine the role of the p38-

907 mediated innate immune signaling pathway in the long-lifespan of the nuo- 6 and isp-1

908 mitochondrial mutants, we crossed the long-lived mitochondrial mutants to worms with

909 mutations in nsy-1, sek-1, pmk-1 and atf-7(gof). In each case, mutation of genes involved in

910 p38-mediated innate immunity signaling markedly reduced the lifespan of the long-lived

911 mitochondrial mutant but had little or no impact on wild-type lifespan. Results are from a

912 minimum of three biological replicates with at least 40 worms per replicate. Statistical analysis

913 on survival plots was performed with log-rank test. $p$-value indicate significance between red

914 and purple lines. The lifespan results for panels $A, B, D, E, F$, and $H$ were performed in a single

915 parallel experiment. Control strains are shown in multiple panels for direct comparison. Raw

916 data and $\mathrm{N}$ for each strain can be found in Table S2.

917

918 
919 Figure 4. p38-mediated innate immune signaling pathway is not activated in long-lived

920 mitochondrial mutants. A. To determine the extent to which the p38-mediated innate

921 immunity pathway is activated in nuo-6 and isp-1 mutants, we measured the ratio of

922 phosphorylated p38/PMK-1 to total p38/PMK-1 by Western blotting. B. There was no

923 difference in the ratio of phosphorylated p38/PMK-1 to total p38/PMK-1 in the long-lived

924 mitochondrial mutants compared to wild-type. Four biological replicates per strain were

925 quantified. See Supplemental Data for complete images of Western blots including loading

926 controls. C. Both long-lived mitochondrial mutants, nuo-6 and isp-1, exhibit markedly decreased

927 food consumption compared to wild-type worms. At least six biological replicates per strain

928 were measured, each including at least 3 technical replicates. D. Deletion of daf-16 significantly

929 increases food consumption in both wild-type and isp-1 mutants. Three biological replicates per

930 strain were measured. E. While disruption of atfs-1 does not affect food intake in wild-type

931 worms, it increases food consumption in nuo- 6 worms back to wild-type. atfs-1 loss of function

932 allele was gk3094. Three biological replicates per strain were measured. F. Constitutive

933 activation of atfs-1 is sufficient to decrease food consumption to the same level as in long-lived

934 mitochondrial mutants. Three biological replicates per strain were measured. atfs-1(lof) allele is 935 gk3094, atfs-1(gof) allele is et17. Error bars indicate SEM. $* * p<0.01, * * * p<0.001$. 
938 Figure 5. DAF-16/FOXO is required for increased resistance to bacterial pathogens in long-

939 lived mitochondrial mutants. A. A deletion of daf-16 completely abolishes the increased

940 resistance to bacterial pathogens in isp-1 mutants. p-value indicates the significance of

941 difference between red and purple lines. Three biological replicates per strain were measured.

942 While daf-16 RNAi effectively decreased the expression of DAF-16 target genes (B, sod-3, dod-3,

$943 m t /-1, f t n-1, i c l-1$, sodh-1), it did not affect the expression of any of the innate immunity genes

944 (C, T24B8.5, K08D8.5, F55G11.8, clec-65, clec-67, dod-22, Y9C9A.8 and C32H11.4). This indicates

945 that DAF-16 is not required for expression of innate immune signaling pathway target genes in

946 isp-1 mutants. daf-16 expression was knocked down using RNAi beginning at the L4 stage of the

947 parental generation. RNA was isolated from six biological replicates at the young adult stage of

948 the experimental generation. RNA from the six biological replicates was pooled for RNA

949 sequencing.

950

951

952 
953 Figure. 6. ATFS-1 is required for increased bacterial pathogen resistance and upregulation of

954 innate immunity genes in long-lived nuo-6 mitochondrial mutants. A. To examine the role of

955 the ATFS-1 transcription factor and the mitochondrial unfolded protein response in the

956 enhanced bacterial pathogen resistance in nuo- 6 mutants, we examined the effect of disrupting

957 atfs-1 in nuo-6 mutants. Loss of atfs-1 completely abolished the increased resistance to

958 bacterial pathogens in nuo- 6 worms. p-value indicates significance of difference between red

959 and purple lines. nuo-6;atfs-1 contains the gk3094 deletion allele. Three biological replicates

960 per strain were measured. B. To examine the role of ATFS-1 in the upregulation of innate

961 immunity genes in nuo-6 mutants, we compared gene expression between nuo-6 and nuo-

962 6;atfs-1 mutants with wild-type worms and atfs-1 mutants as controls. Deletion of atfs-1

963 significantly decreased the expression of genes involved in innate immunity in nuo- 6 worms,

964 but did not decrease the expression of these genes in wild-type worms. Gene expression

965 changes were determined by RNA sequencing of six biological replicates of each genotype.

966 Results represent counts per million (CPM) expressed as a percentage of wild-type. Worms in a

967 wild-type background (control) are shown with white bars, while worms with atfs-1 deletion

968 are shown with blue bars. Error bars indicate SEM. ${ }^{*} p<0.01, * * * p<0.001$. 


\section{Figure. 7. Constitutive activation of mitochondrial unfolded protein response results in}

972 upregulation of innate immunity genes. A. Examination of mRNA levels of genes involved in

973 innate immunity in two constitutively active atfs-1 mutants (et15 and et17) revealed that innate

974 immunity genes are upregulated when ATFS-1 is activated. This indicates that activation of the

975 mitochondrial unfolded protein response can cause activation of genes that are regulated by

976 the p38-mediated innate immune pathway. Gene expression changes were determined by RNA

977 sequencing of six biological replicates of each genotype. Results represent counts per million

978 (CPM) expressed as a percentage of wild-type. B. Constitutive activation of ATFS-1 does not

979 increase activation of PMK-1/p38 as measured by the ratio of phospho-p38 to total p38 using

980 Western blotting. atfs-1(gof) is et17 allele. atfs-1(lof) is $g k 3094$ allele. Protein levels were

981 quantified for four biological replicates. See Supplemental Data for complete images of

982 Western blots including loading controls. Error bars indicate SEM. ${ }^{*} p<0.05 * * p<0.01$,

$* * * p<0.001$.

984

985

986 
987 Figure 8. Mild impairment of mitochondrial function increases bacterial pathogen resistance

988 and lifespan. Impairment of mitochondrial function through mutation of nuo- 6 or isp-1, which

989 affect the mitochondrial electron transport chain, leads to the activation of the mitochondrial

990 unfolded protein response (mitoUPR) and nuclear localization of DAF-16. The mitoUPR

991 transcription factor ATFS-1 can bind directly to innate immunity genes to increase their

992 expression. Activation of ATFS-1 is also sufficient to decrease food consumption, which results

993 in decreased signaling through the p38-mediated innate immune signaling pathway. This

994 signaling pathway terminates with the ATF-7 transcription factor that can bind to the same

995 genes as ATFS-1. ATFS-1 also acts to facilitate the nuclear localization of DAF-16, which

996 modulates the expression of a separate set of genes that promotes survival of bacterial

997 pathogens and longevity. DAF-16 acts to decrease food intake thereby decreasing signaling

998 through the p38-mediated innate immune signaling pathway. DAF-16, ATFS-1, and all of the

999 components of the p38-mediated innate immune signaling pathway (NSY-1, SEK-1, PMK-1 and

1000 ATF-7) are required for the increased resistance to bacterial pathogens and increased lifespan

1001 in nuo-6 and isp-1 mutants. 
bioRxiv preprint doi: https://doi.org/10.1101/2021.03.31.437812; this version posted April 1, 2021. The copyright holder for this preprint (which A was not certified by peer review) is the author/funder, who has granted bioRxiv a license to display the preprint in perpetuity. It is made
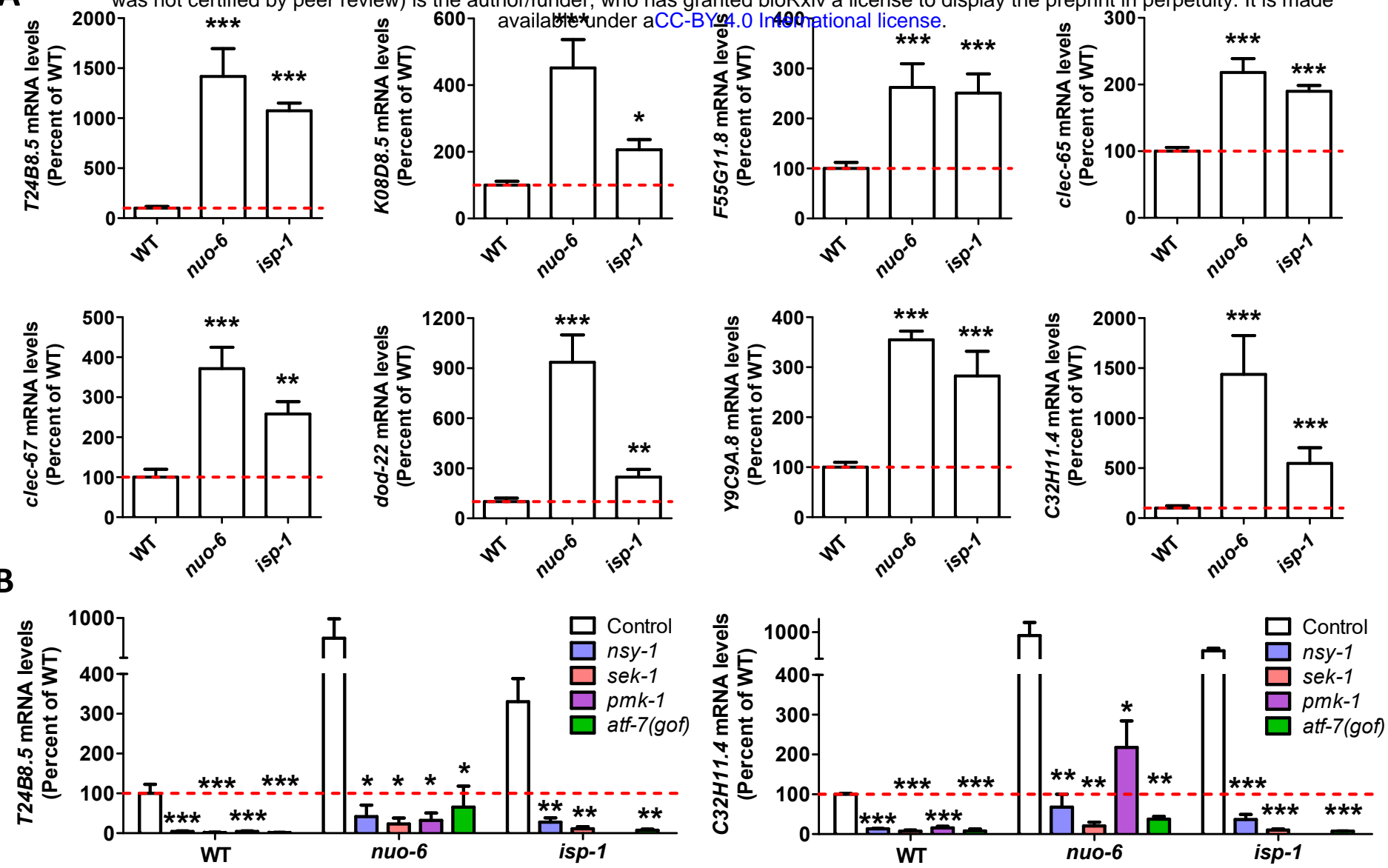

C
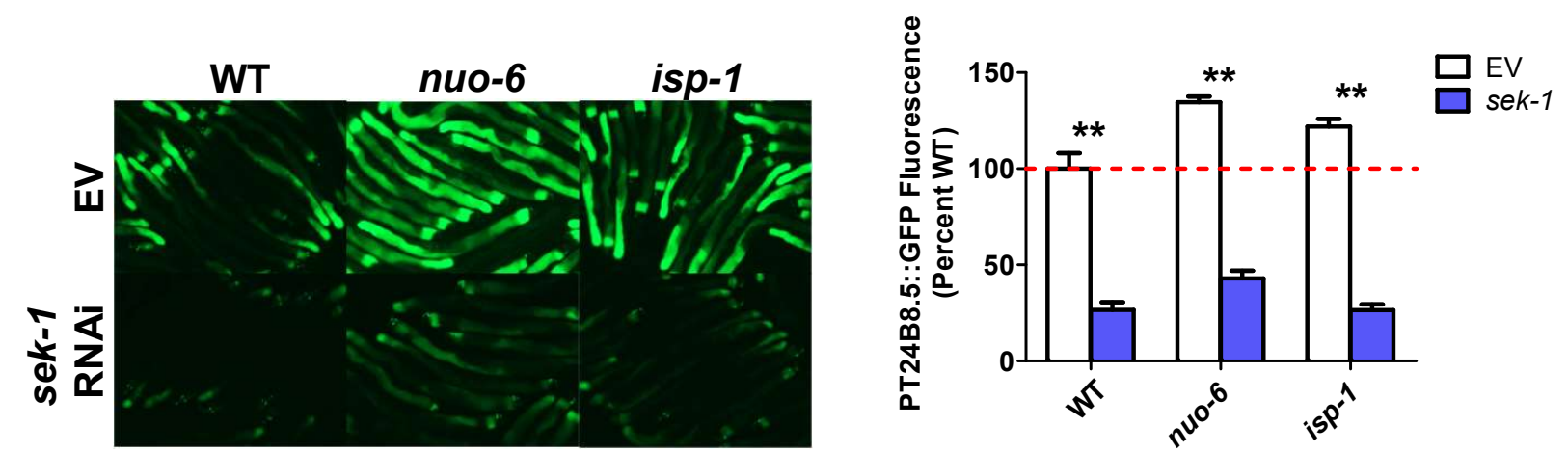

D
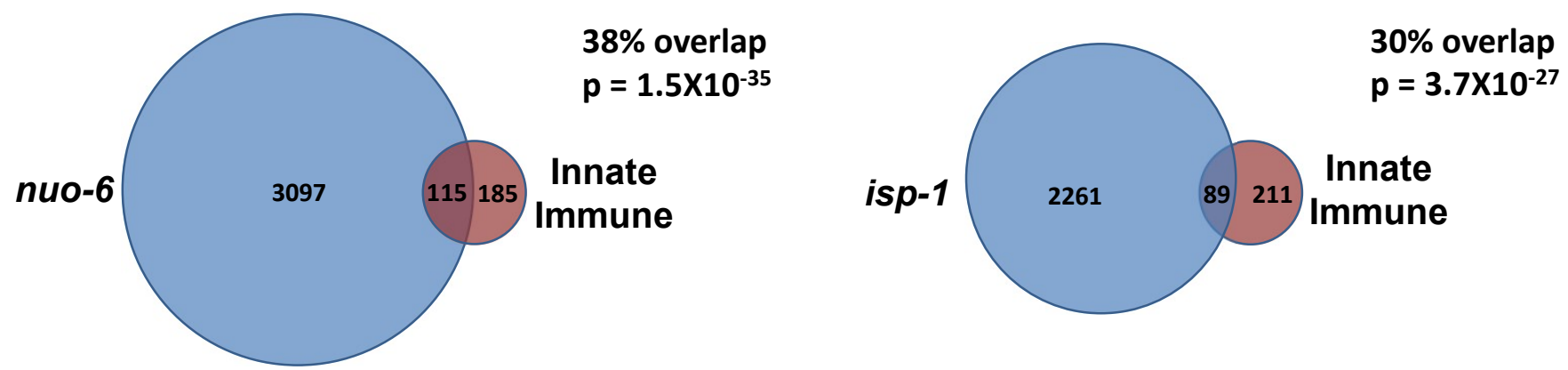

Figure 1. Innate immunity genes are upregulated in long-lived mitochondrial mutants. 
A

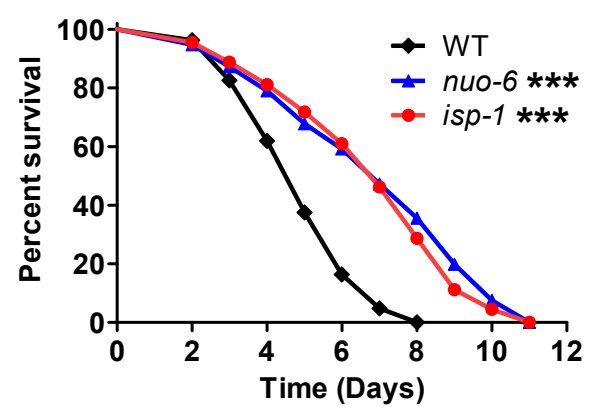

C

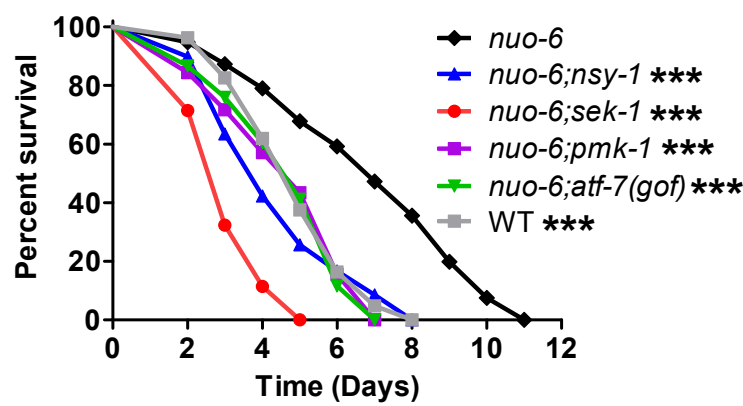

B

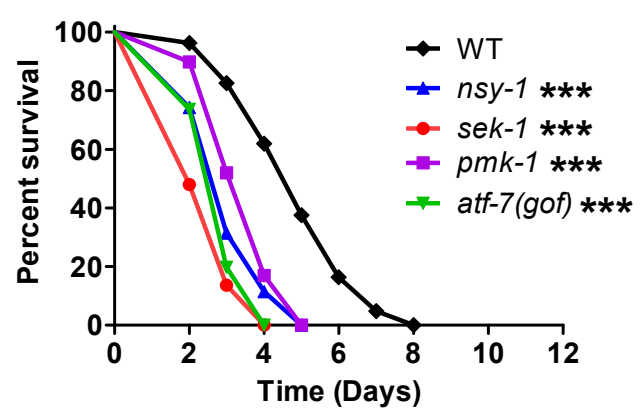

D

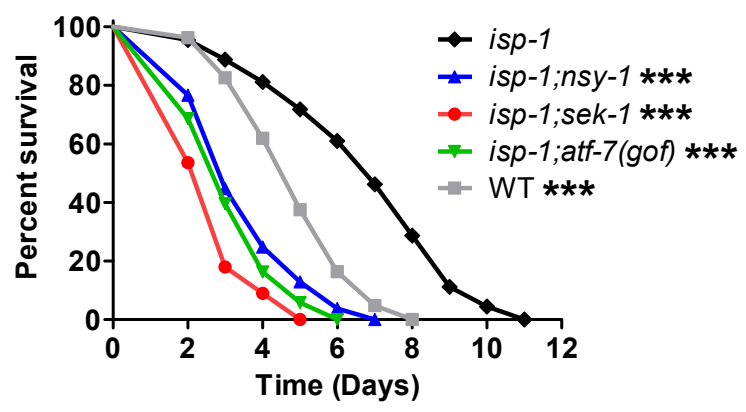

Figure 2. Long-lived mitochondrial mutants exhibit increased resistance to bacterial pathogens that is dependent on presence of p38-mediated innate immune signaling pathway. 
A

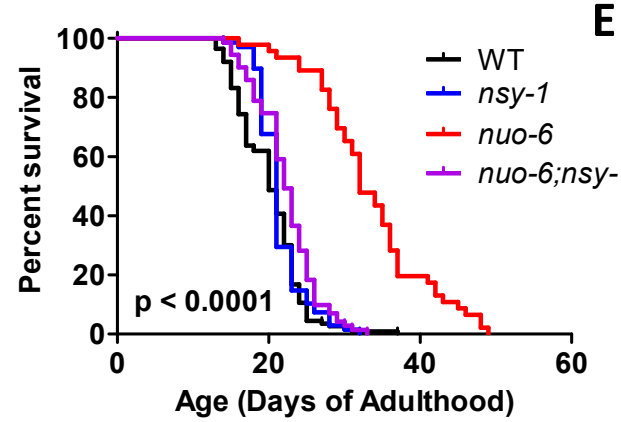

B

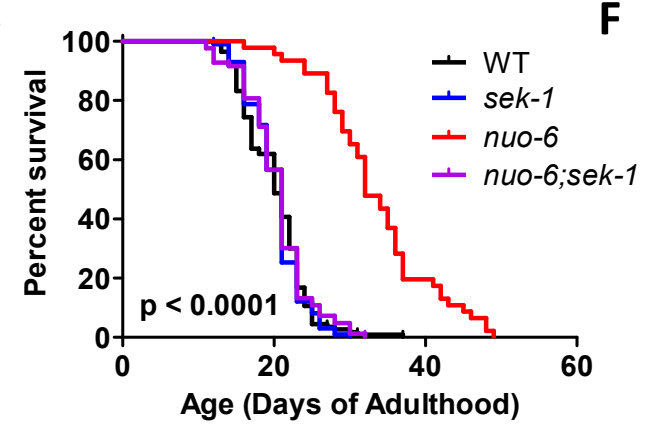

C

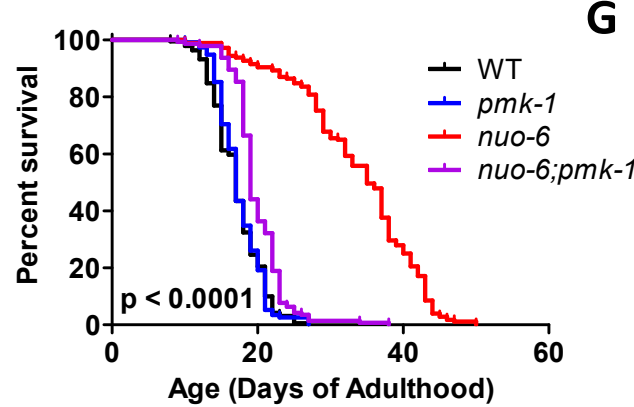

D

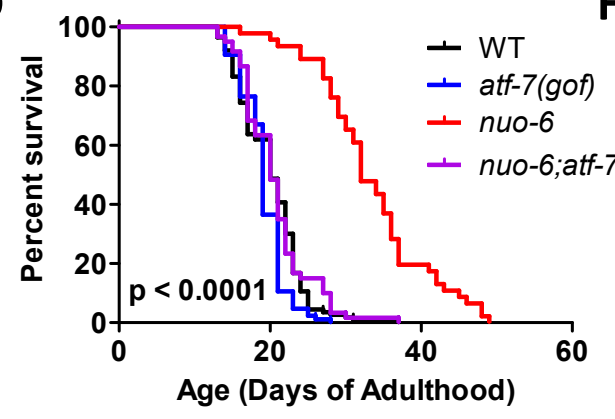

E

$\mathbf{F}$

H
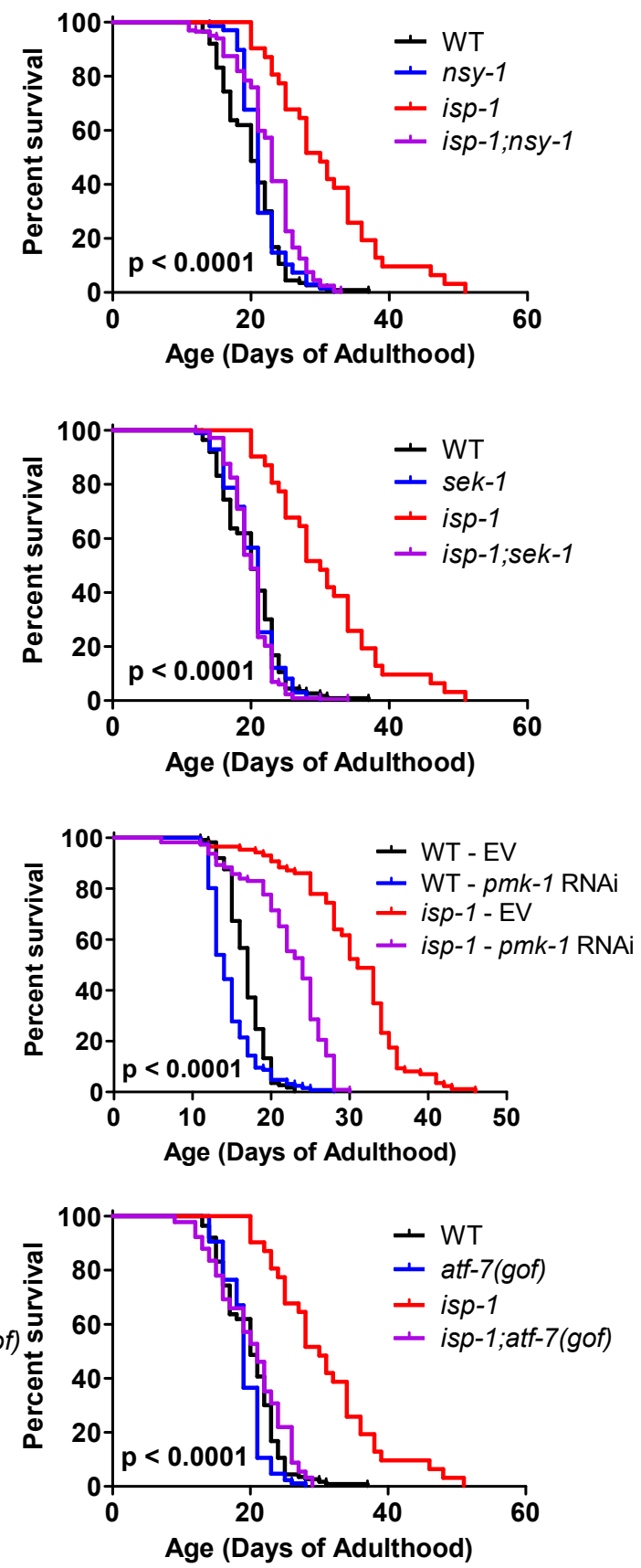

Figure 3. Disruption of the p38-mediated innate immune signaling pathway markedly ablates extended longevity of long-lived mitochondrial mutants. 
A
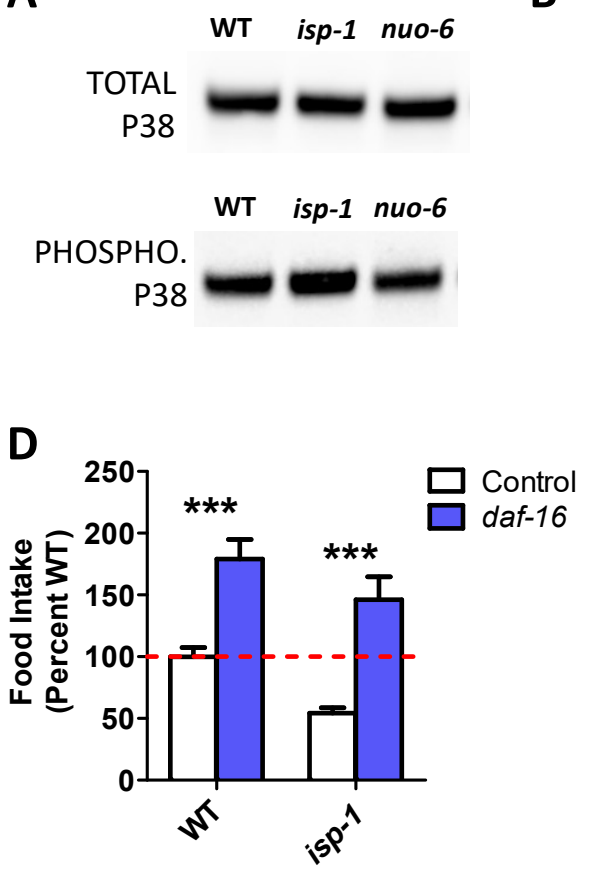

B
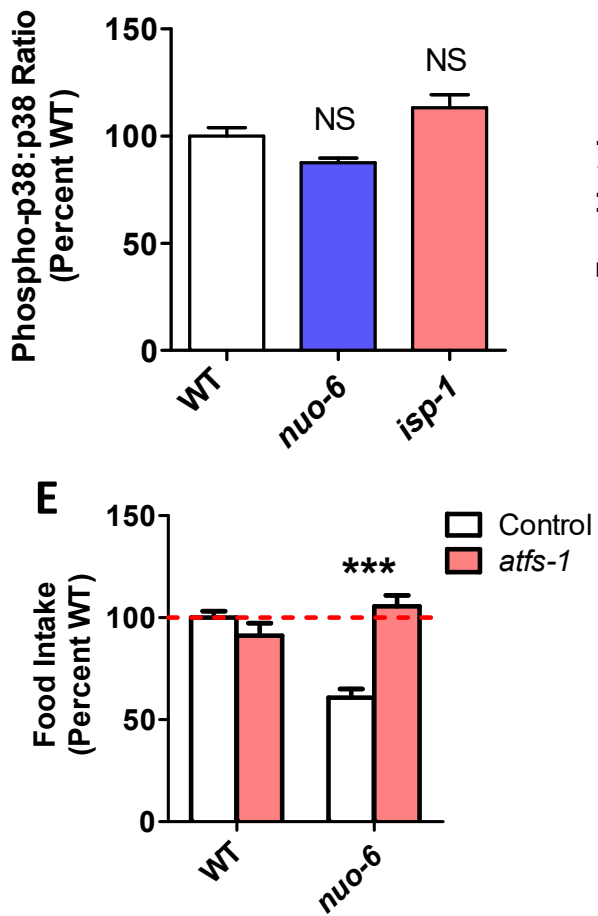
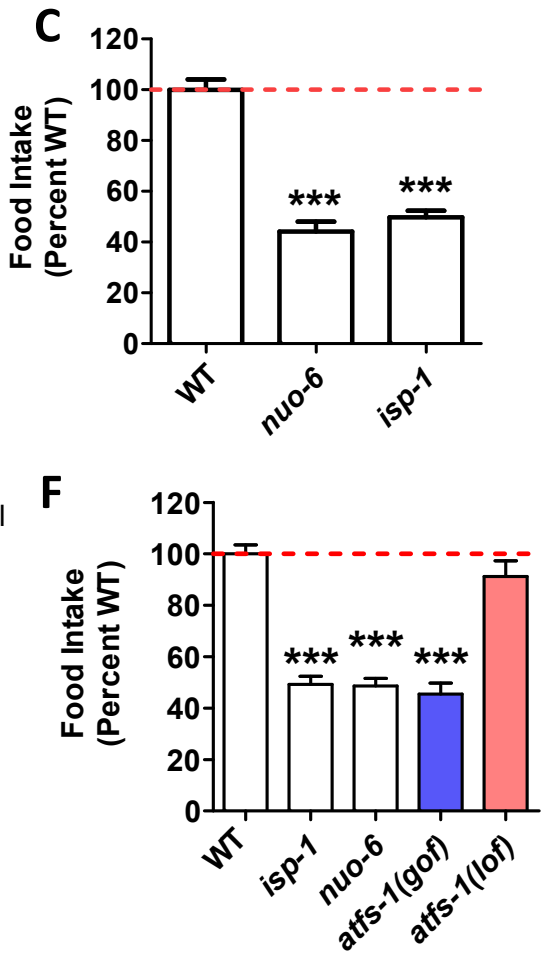

Figure 4. p38-mediated innate immune signaling pathway is not activated in long-lived mitochondrial mutants. 
A

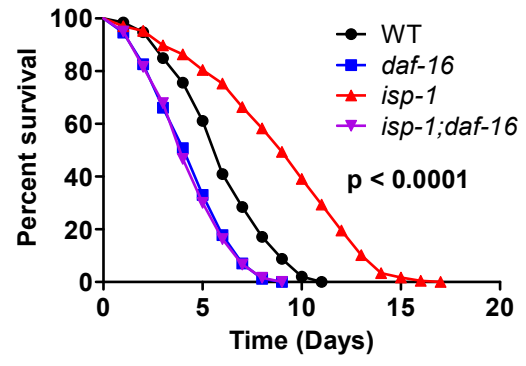

B

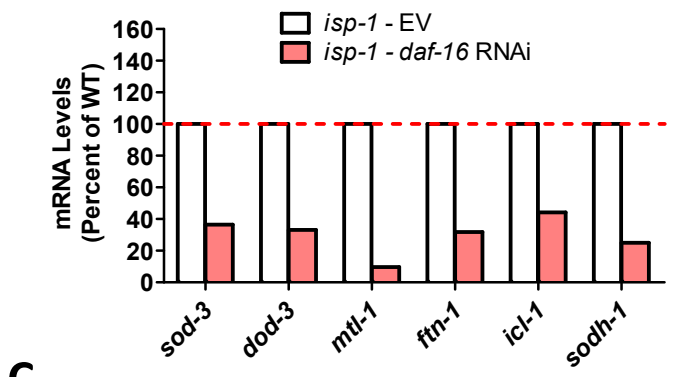

C

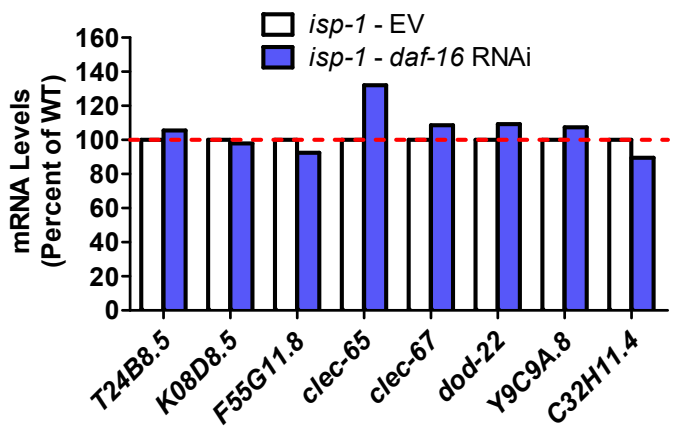

Figure 5. DAF-16/FOXO is required for increased resistance to bacterial pathogens in long-lived mitochondrial mutants. 
A

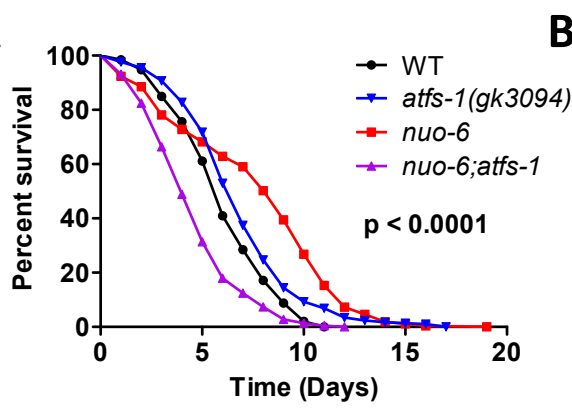
available under aCC-BY 4.0 International license.
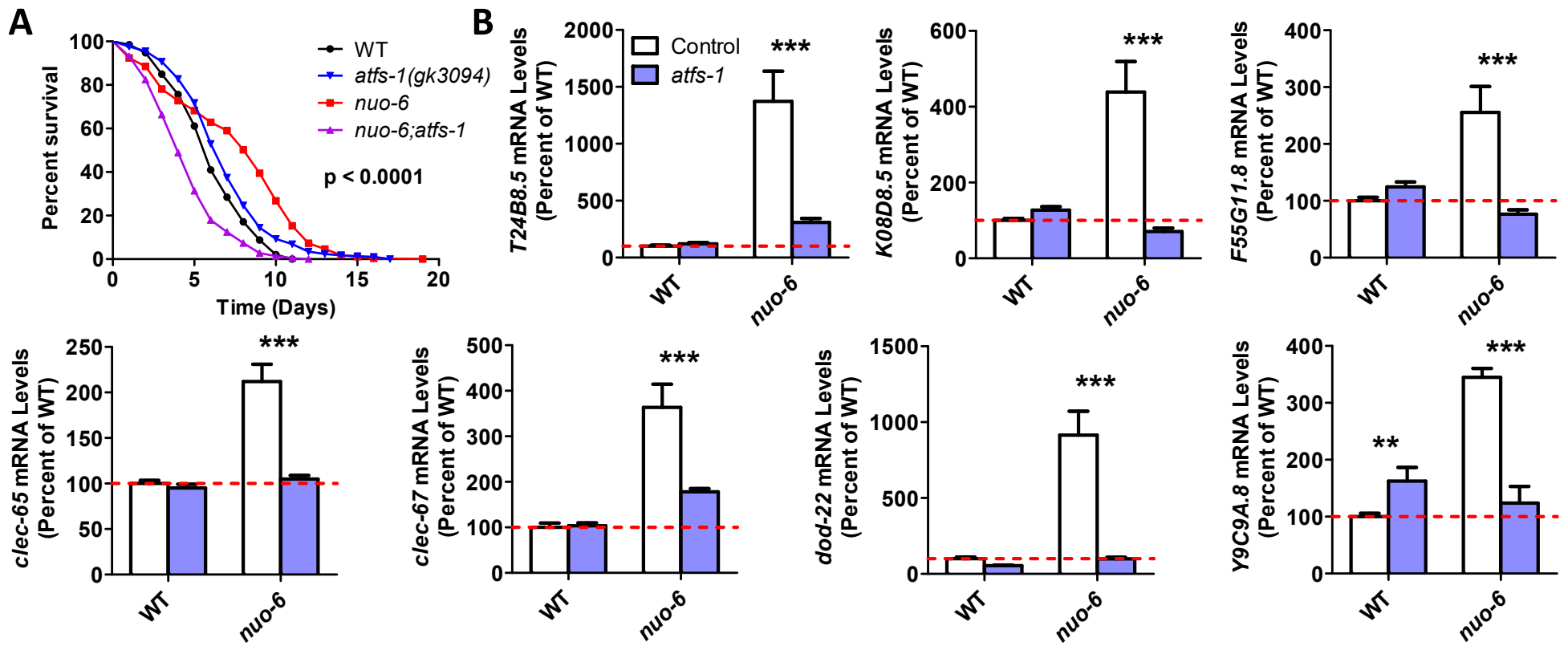

Figure 6. ATFS-1 is required for increased bacterial pathogen resistance and upregulation of innate immunity genes in long-lived nuo-6 mitochondrial mutants. 
bioRxiv preprint doi: https://doi.org/10.1101/2021.03.31.437812; this version posted April 1, 2021. The copyright holder for this preprint (which was not certified by peer review) is the author/funder, who has granted bioRxiv a license to display the preprint in perpetuity. It is made

A
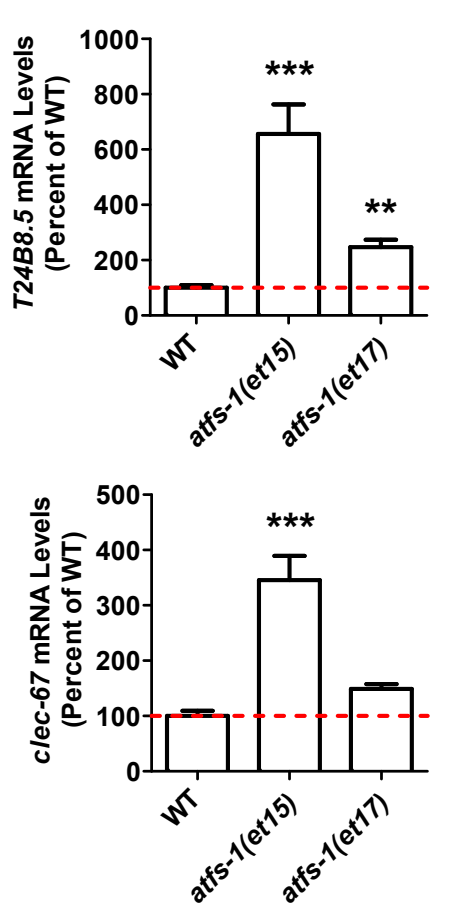

B

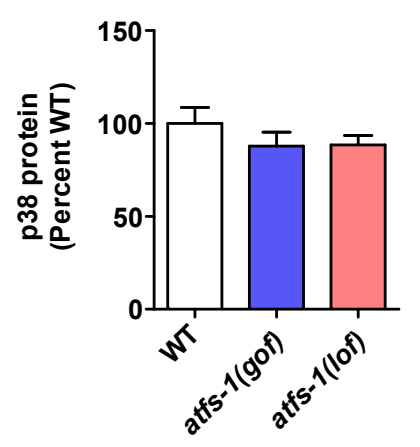

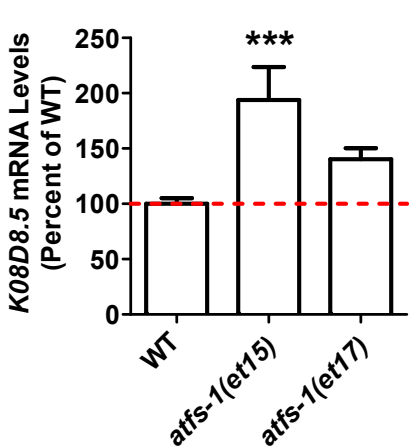
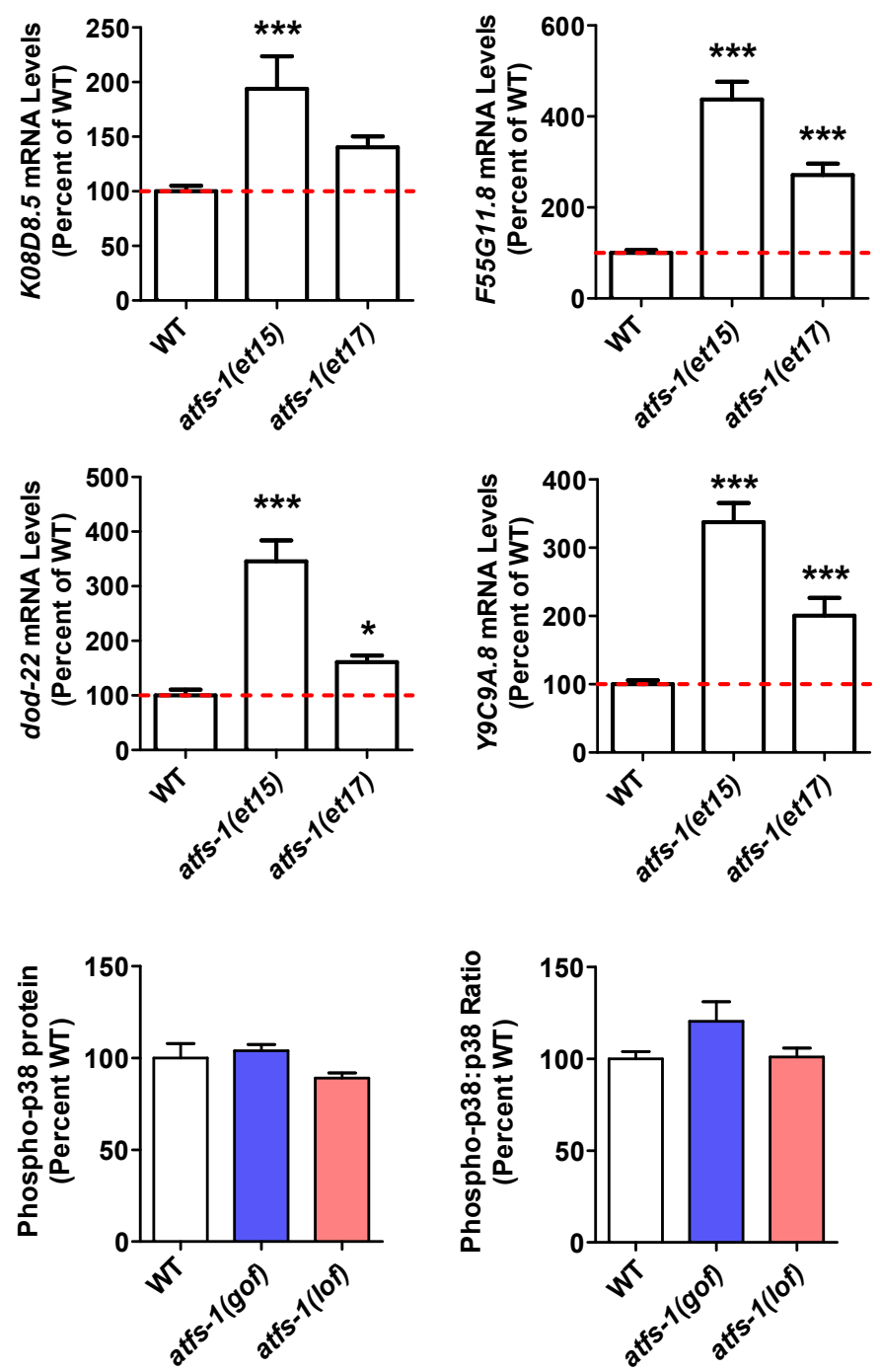
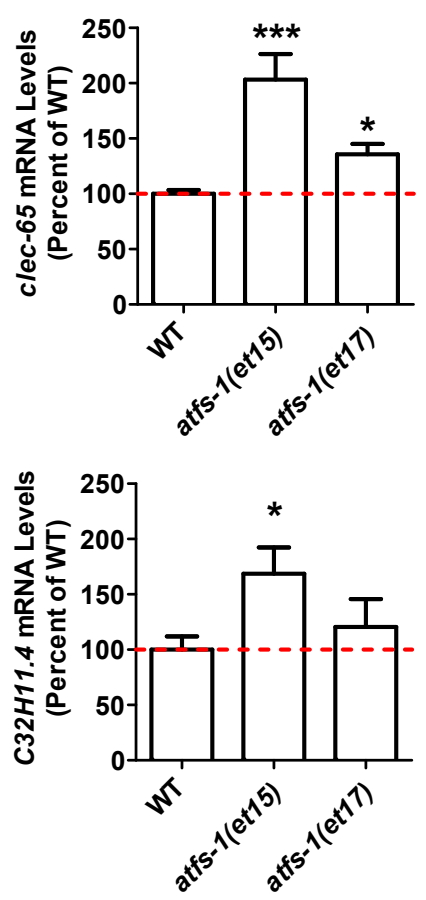

Figure 7. Constitutive activation of mitochondrial unfolded protein response results in upregulation of innate immunity genes. 


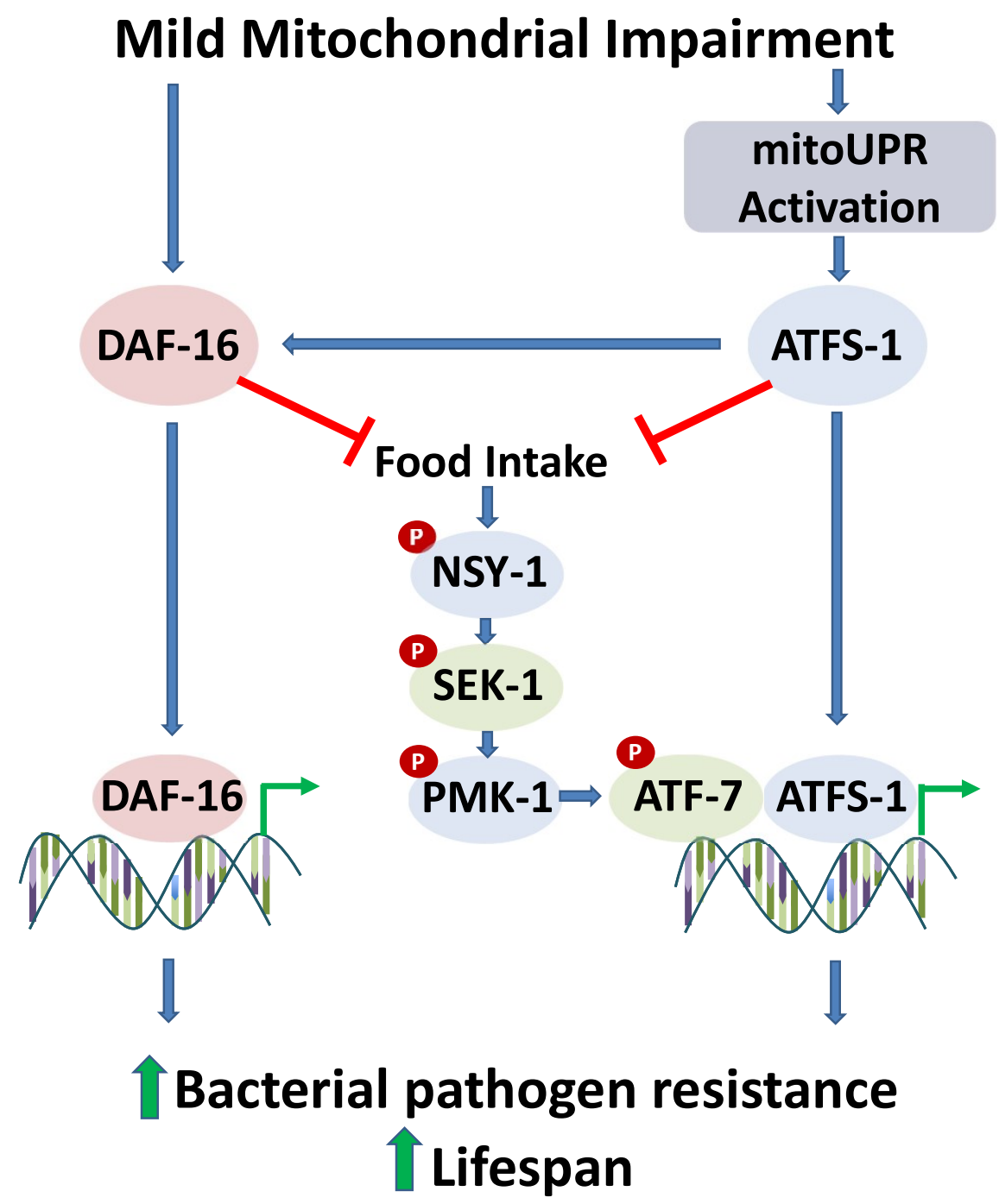

Figure 8. Mild impairment of mitochondrial function increases bacterial pathogen resistance and lifespan. 


\section{Supplementary Figures for:}

\section{Mild mitochondrial impairment increases longevity and pathogen resistance through ATFS-1-driven activation of p38-regulated innate immunity}

Juliane C. Campos\#, Ziyun Wü, Paige D. Rudich, Sonja K. Soo, Meeta Mistry, Julio C.B. Ferreira, T. Keith Blackwell, Jeremy M. Van Raamsdonk

\section{Corresponding authors:}

T. Keith Blackwell

Email: keith.blackwell@joslin.harvard.edu

Jeremy M. Van Raamsdonk

Email: jeremy.vanraamsdonk@mcgill.ca

This PDF file includes:

Figures S1 to S12

Supplementary data: Western blot image

Other supplementary materials for this manuscript include the following:

Tables S1 to S3 


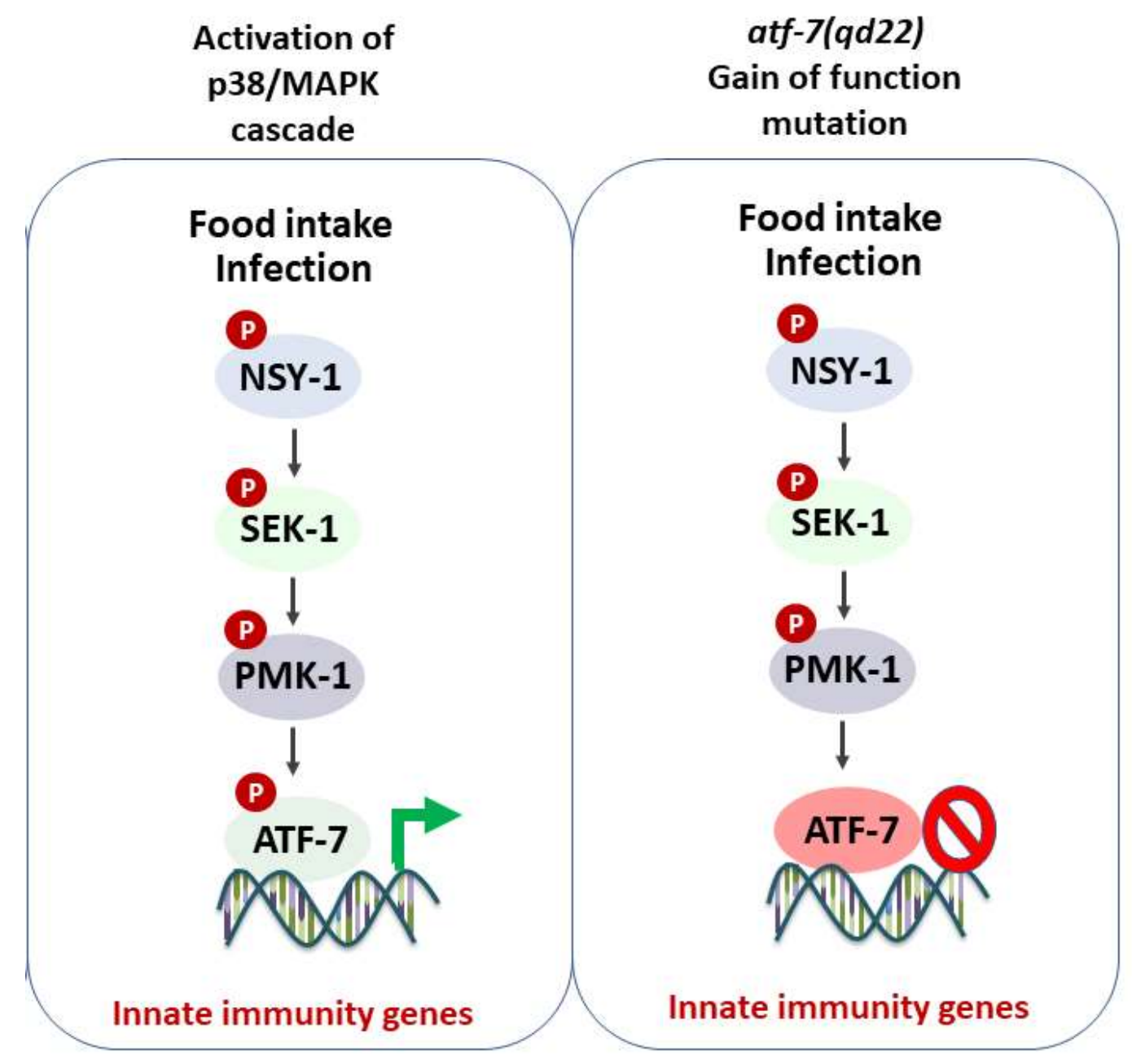

Figure S1. Overview of p38-mediated innate immune signaling pathway. The p38-mediated innate immune signaling pathway is a MAPK signaling pathway. Exposure to bacterial pathogens or increased food intake results in the activation of this pathway through the phosphorylation of NSY-1/ASK1 (MAPK kinase kinase). NSY-1 then phosphorylates SEK-1/MKK3/MKK6 (MAPK kinase), which phosphorylates PMK-1/p38 (MAPK), which phosphorylates the transcription factor ATF-7/ATF2/ATF7/CREB5. Under normal conditions, ATF-7 acts as a repressor inhibiting the expression of innate immunity genes. When ATF-7 is phosphorylated by PMK-1, it becomes an activator promoting the expression of innate immunity genes. The $q d 22$ mutation prevents phosphorylation of ATF-7 by PMK-1. As a result, ATF-7 with the $q d 22$ mutation acts as a constitutive repressor even in the presence of bacterial pathogens. 
A

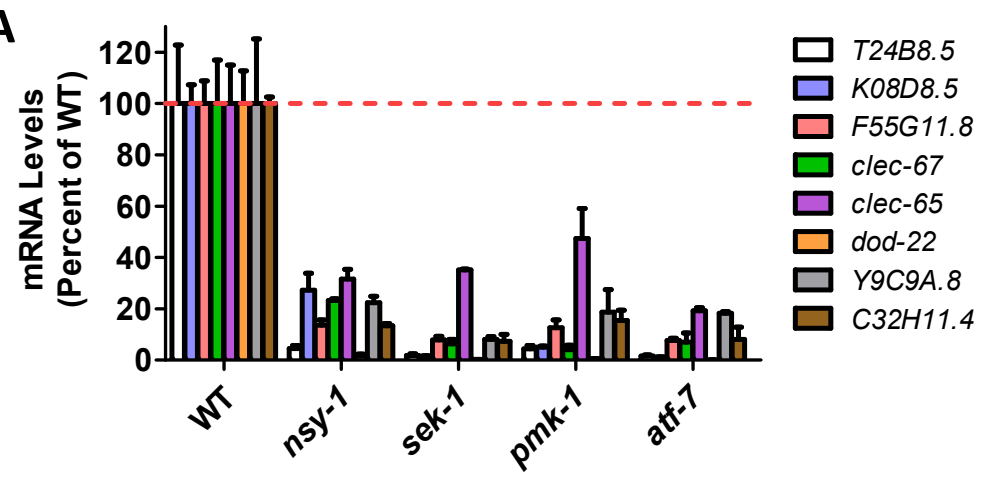

B

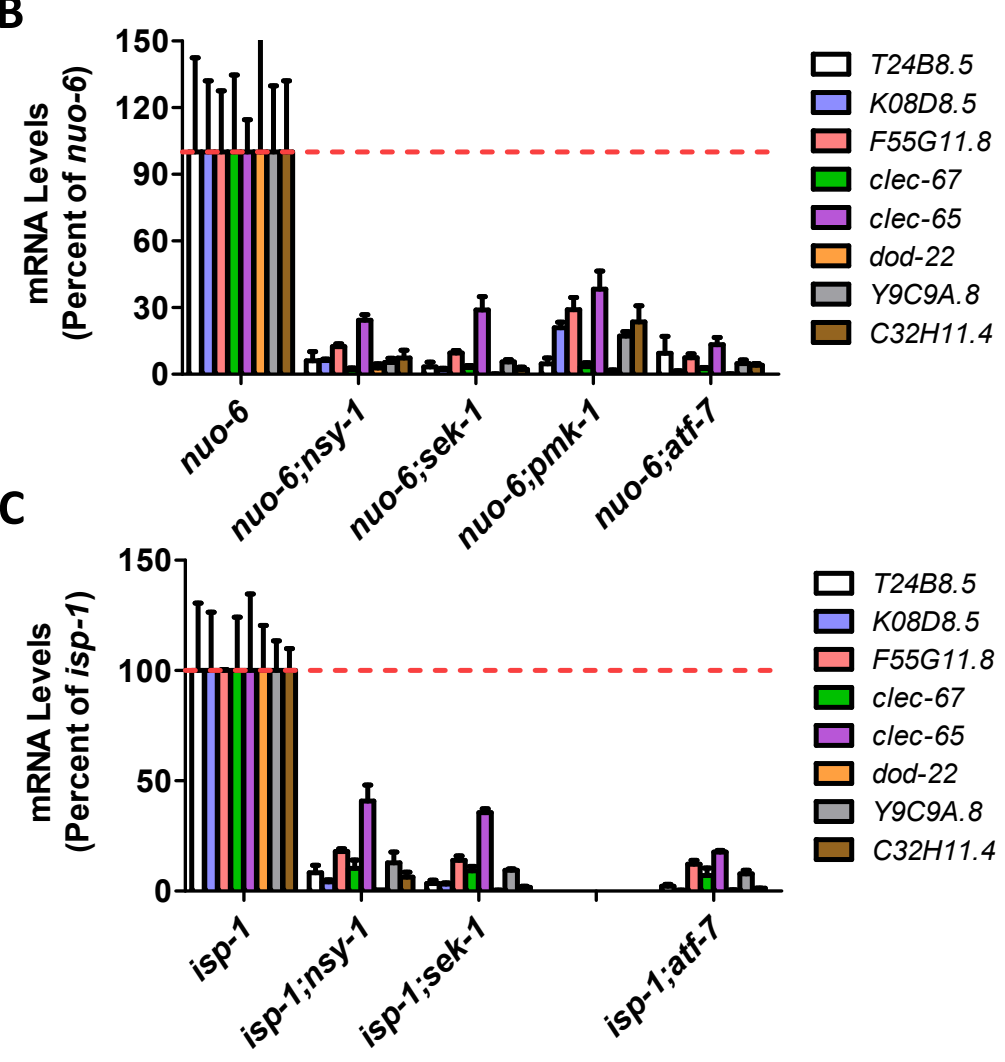

Figure S2. Upregulation of innate immunity genes in long-lived mitochondrial mutants requires the p38mediated innate immune signaling pathway. Mutation of genes involved in the p38-mediated innate immune signaling pathway (nsy-1, sek-1, pmk-1, atfs-7(gof)) decrease the expression of genes involved in innate immunity in wild-type (A), nuo-6 (B), and isp-1 (C) worms. Gene expression was determined by quantitative real-time RT-PCR on three biological replicates of pre-fertile young adult worms. Error bars indicate SEM. All differences from control are significant $p<0.05$. 


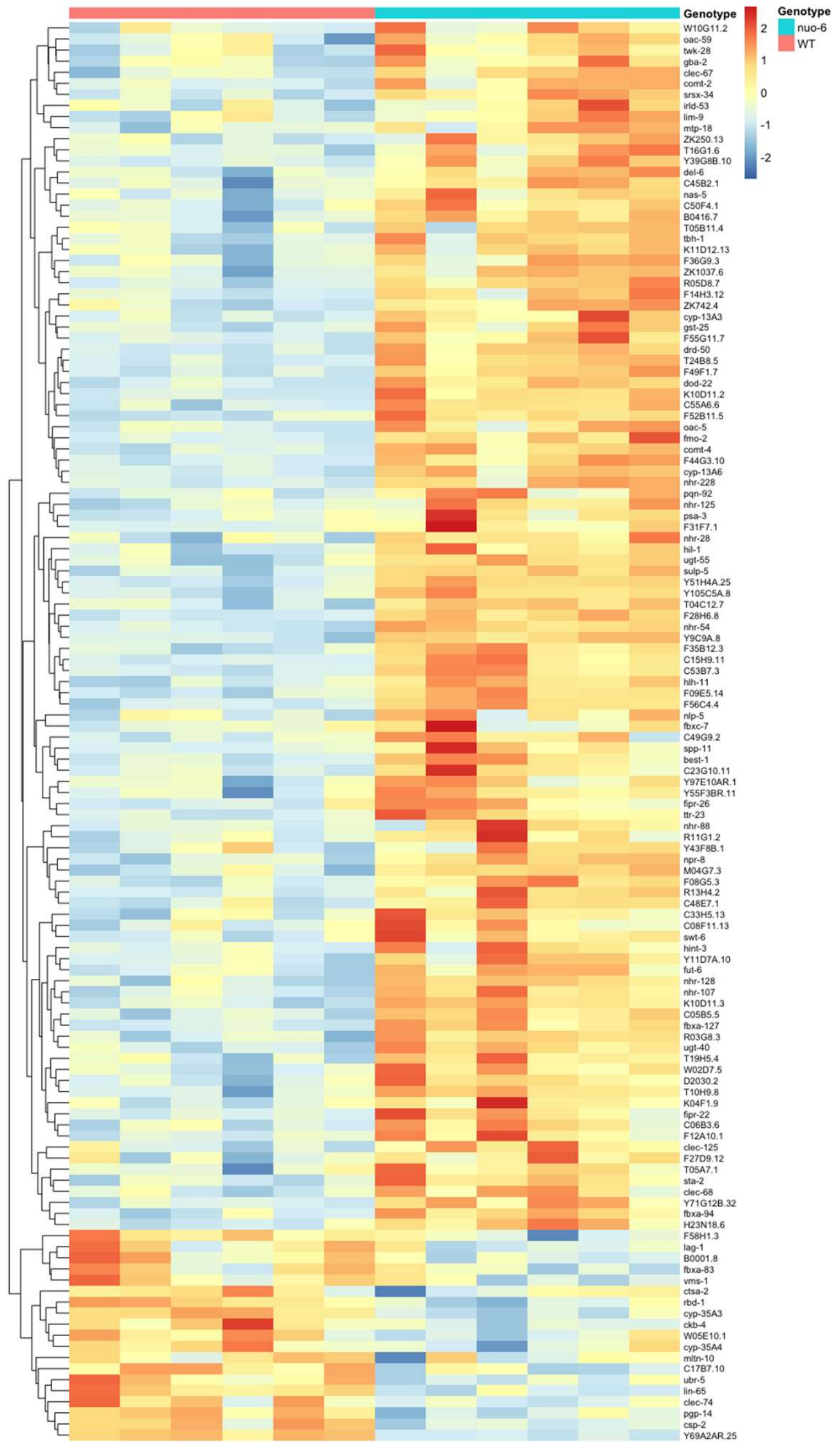

Figure S3. Genes that are upregulated by activation of the p38-mediated innate immune pathway are primarily upregulated in the long-lived mitochondrial mutant nuo-6. This heatmap includes genes that are upregulated by exposure to the bacteria pathogen $P$. aeruginosa PA14 in a PMK-1- and ATF-7-dependent manner and for which the expression is significantly changed in nuo-6 mutants. 

available under aCC-BY 4.0 International license.

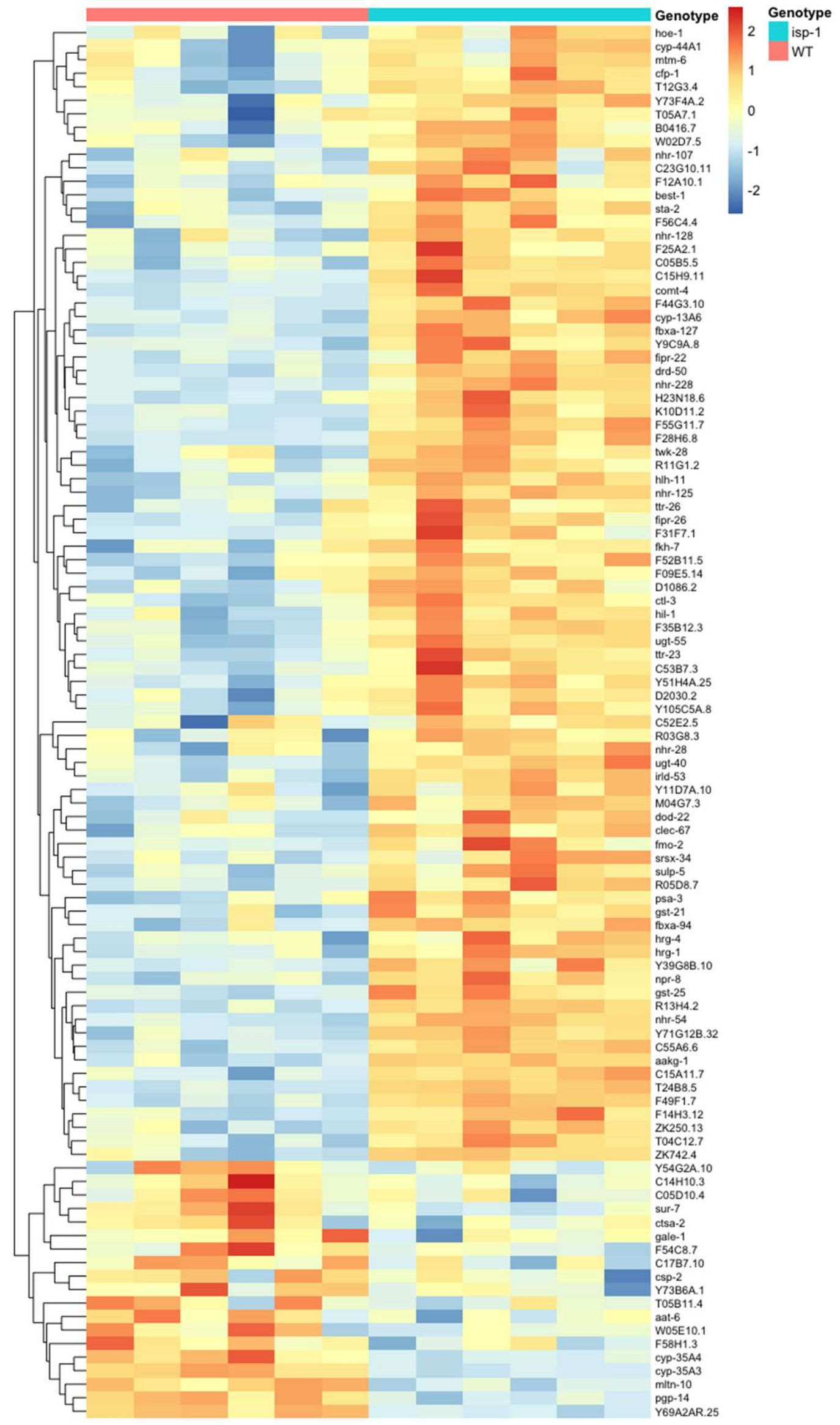

Figure S4. Genes that are upregulated by activation of the p38-mediated innate immune pathway are primarily upregulated in the long-lived mitochondrial mutant isp-1. This heatmap includes genes that are upregulated by exposure to the bacteria pathogen P. aeruginosa PA14 in a PMK-1- and ATF-7dependent manner and for which the expression is significantly changed in isp-1 mutants. 


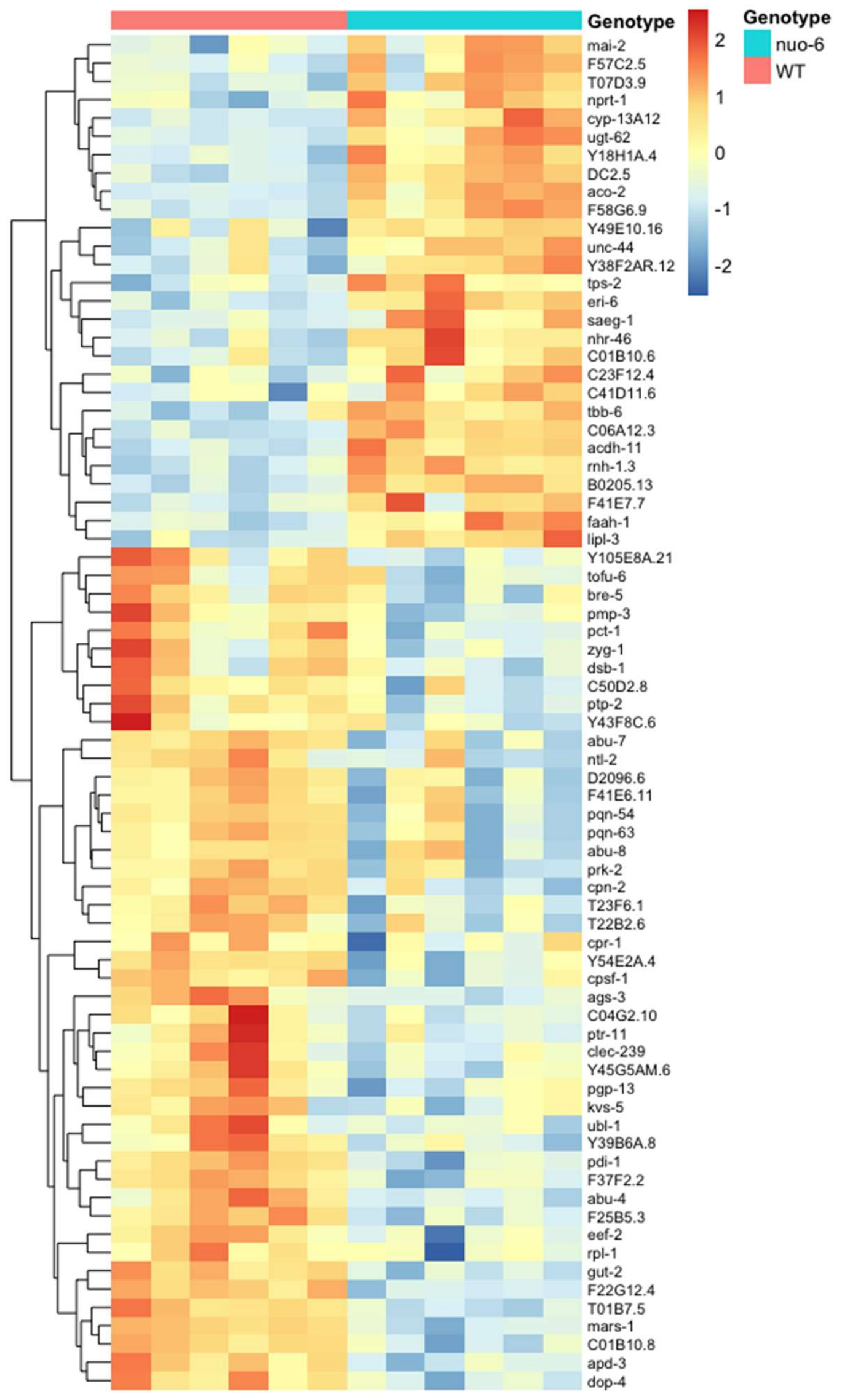

Figure S5. Expression of genes that are downregulated by activation of the p38-mediated innate immune pathway is altered in the long-lived mitochondrial mutant nuo-6. This heatmap includes genes that are downregulated by exposure to the bacteria pathogen $P$. aeruginosa PA14 in a PMK-1and ATF-7--dependent manner and for which the expression is significantly changed in nuo- 6 mutants. 


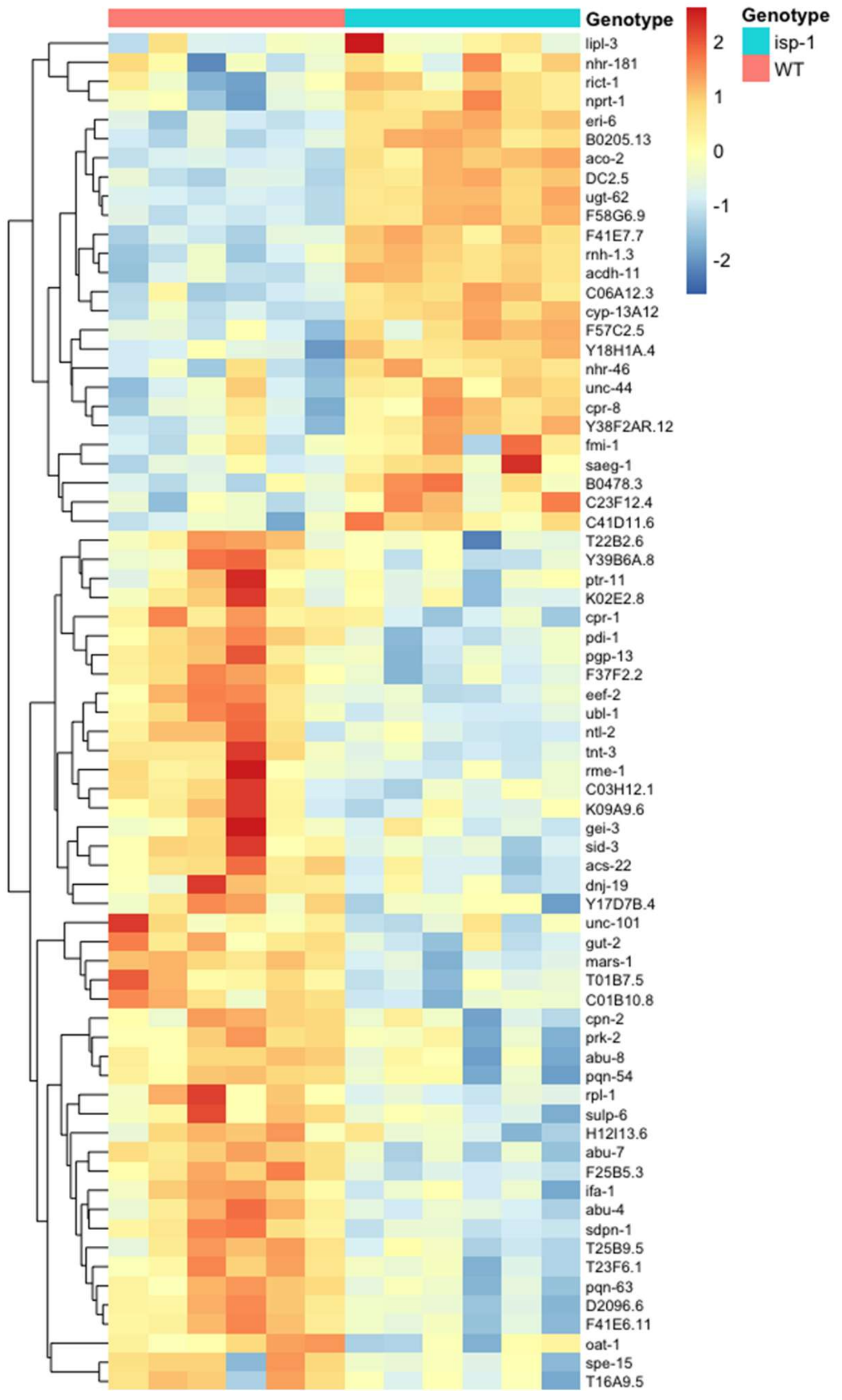

Figure S6. Expression of genes that are downregulated by activation of the p38-mediated innate immune pathway is altered in the long-lived mitochondrial mutant isp-1. This heatmap includes genes that are downregulated by exposure to the bacteria pathogen $P$. aeruginosa PA14 in a PMK-1and ATF-7--dependent manner and for which the expression is significantly changed in isp-1 mutants. 

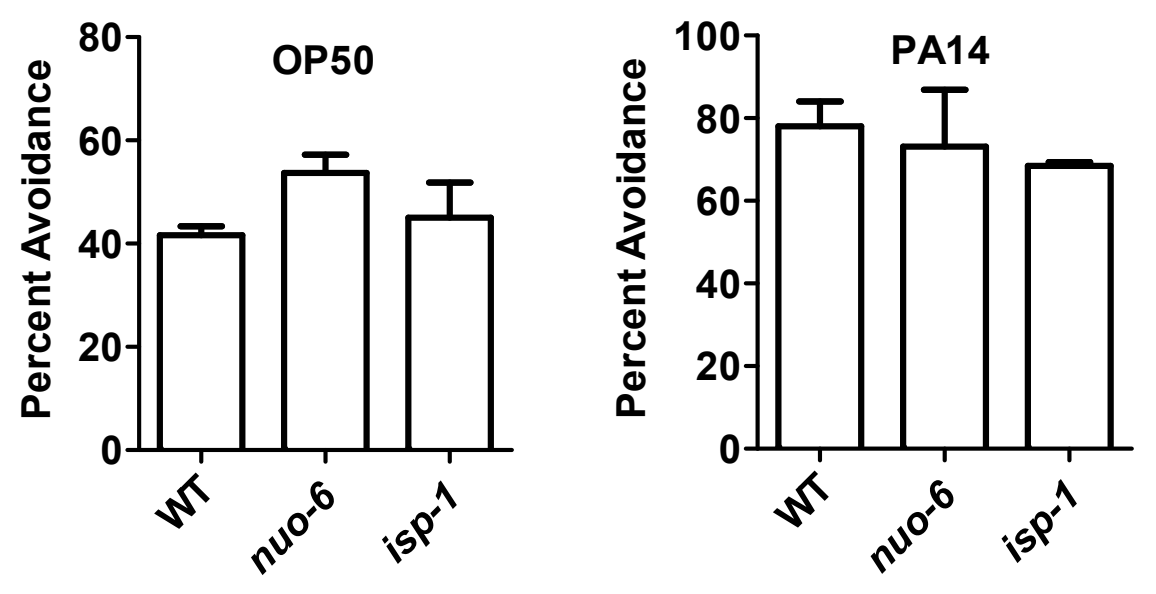

Figure S7. Increased resistance to bacterial pathogens does not result from bacterial avoidance. To explore the mechanisms underlying the increased resistance to bacterial pathogens in nuo- 6 and isp-1 mutants, bacterial avoidance and food consumption were examined. There was no significant difference in bacterial avoidance between wild-type worms and nuo-6 or isp-1 mutants on OP50 bacteria (A) or P. aeruginosa (B). Three biological replicates per strain were quantified. Error bars indicate SEM. 


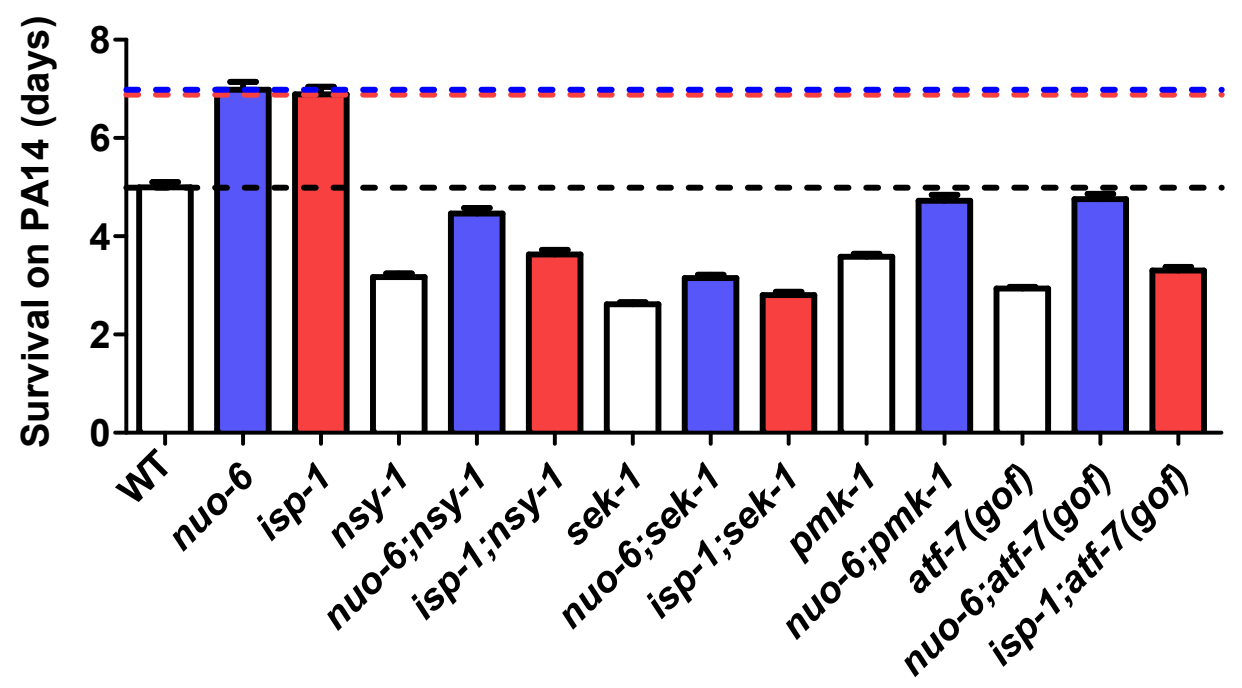

Figure S8. Disruption of p38-mediated innate immune signaling pathway decreases resistance to bacterial pathogens. Resistance to bacterial pathogens was tested by exposing worms to Pseudomonas aeruginosa strain PA14 in a slow kill assay. Long-lived mitochondrial mutants, nuo- 6 and isp-1, have increased survival on pathogenic PA14 bacteria. Mutations affecting genes involved in the p38-mediated innate immune signaling pathway including nsy-1, sek-1, pmk-1 and atf-7(gof) cause decreased resistance to bacterial pathogens in wild-type (white bars), nuo-6 (blue bars) and isp-1 (red bars) worms. Black dotted line indicates wild-type survival. Blue dotted line indicates nuo- 6 survival. Red dotted line indicates isp-1 survival. Survival is measured as the number of days from exposure to PA14 (day 3 of adulthood) until death. Error bars indicate SEM of survival of individual animals. This bar graph is a summary of data shown in Figure 2 to facilitate comparison across all strains. Raw data and total $\mathbf{N}$ per strain are provided in Table $\mathbf{S 2}$. 


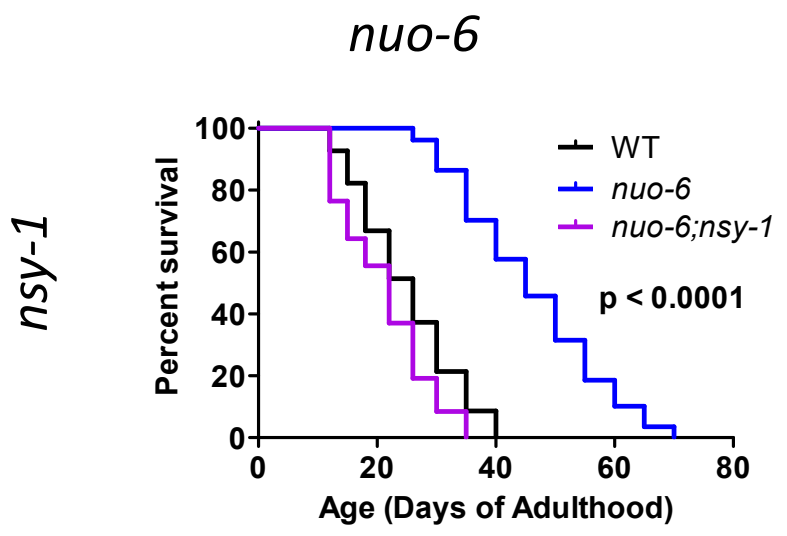

isp-1
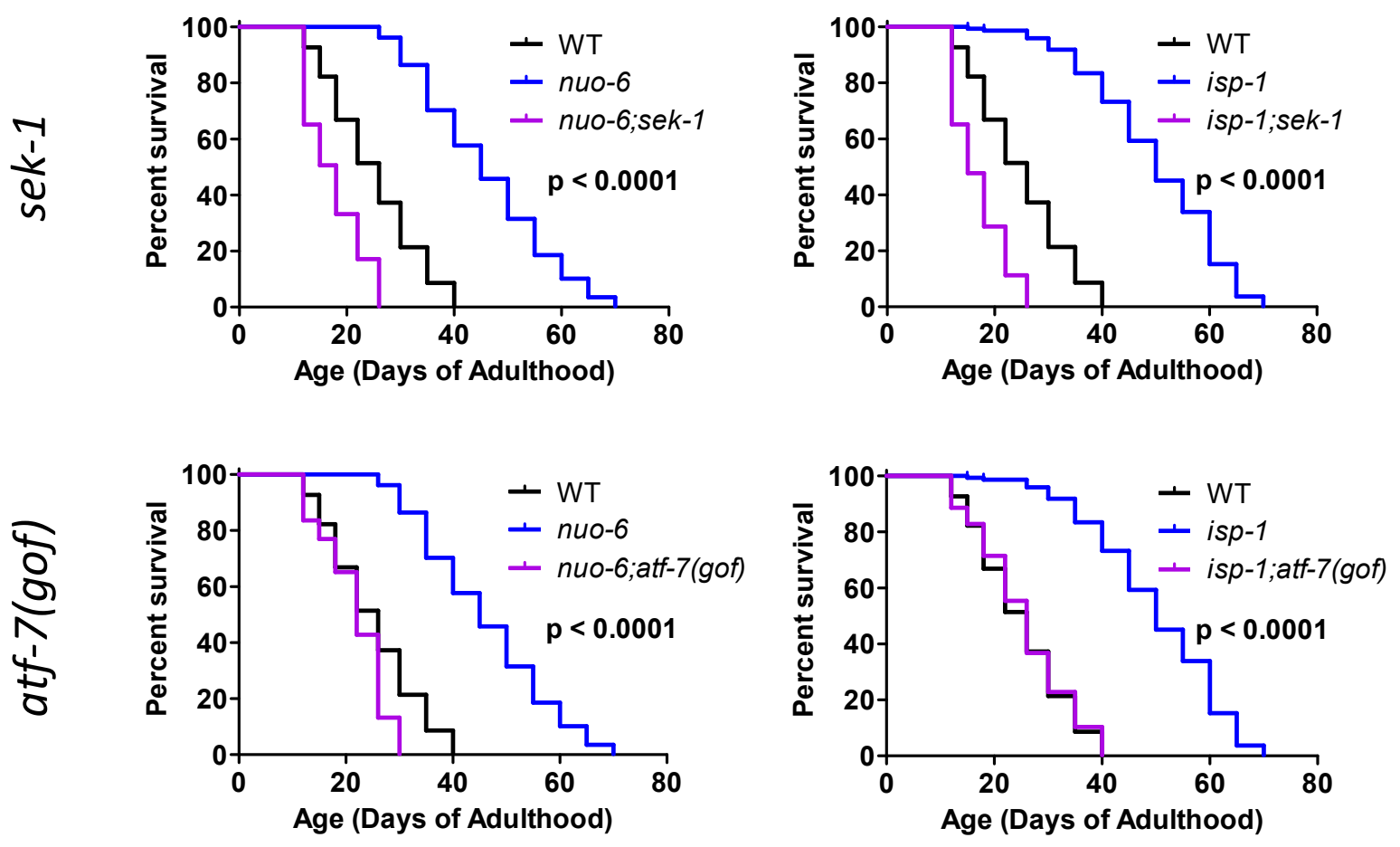

Figure S9. Disruption of genes involved in the p38-mediated innate immune signaling pathway abolishes the extended longevity of long-lived mitochondrial mutants independently of bacterial proliferation. Quantification of nuo- 6 and isp-1 lifespan on non-proliferating bacteria revealed that their long lifespan is independent of bacterial proliferation. Similarly, lifespan extension in nuo- 6 and isp-1 mutants is completely dependent on having a function p38-mediated innate immune signaling pathways as deletion of $n s y-1$, sek1, or atf-7(gof) completely prevented the increase in lifespan in long-lived nuo-6 and isp-1 mutants. Lifespans were performed in liquid culture with worms fed ad libitum. Bacteria proliferation was prevented through treatment with cold and antibiotics. Statistical analysis on survival plots was performed with logrank test. $p$-value indicates significance of difference between blue and purple lines. All strains were tested in a single parallel experiment. Control strains are shown in multiple panels for direct comparison. Raw data and total $\mathbf{N}$ per strain can be found in Table $\mathbf{S 2}$. 


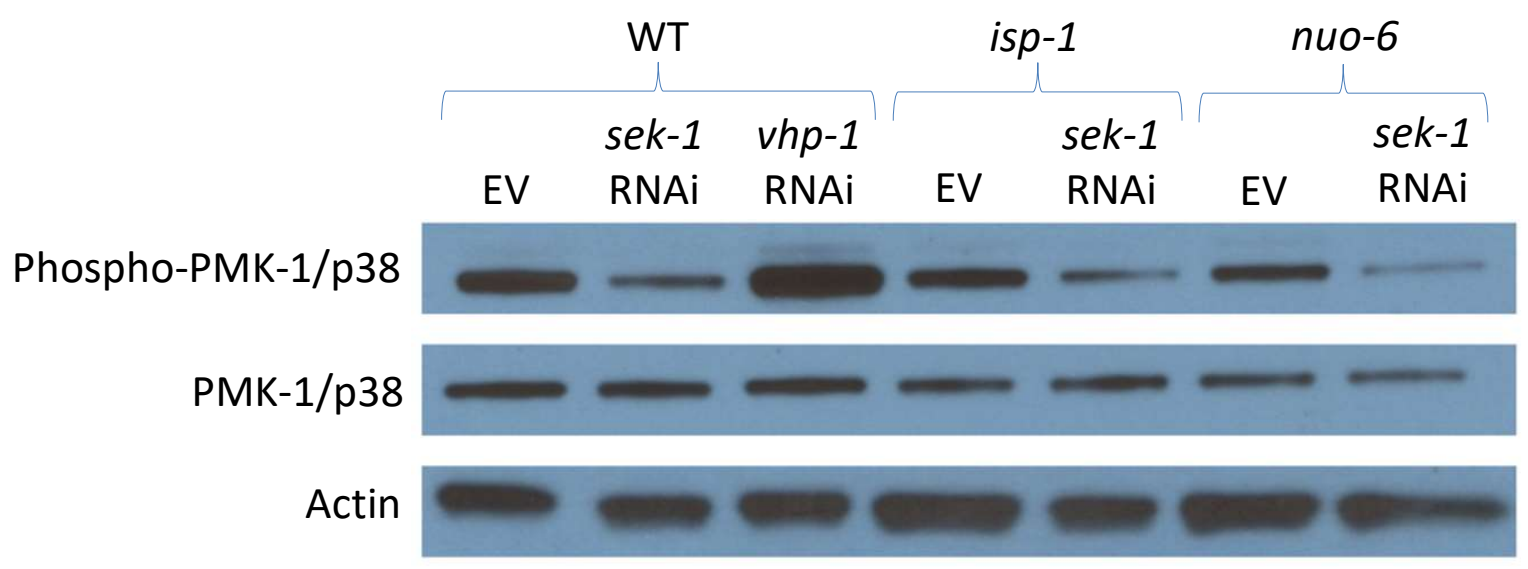

Figure S10. Proportion of activated PMK-1/p38 is not increased in nuo- 6 and isp-1 worms. Activation of PMK-1/p38 was measured by levels of phosphorylated p38/PMK-1 compared to total levels of PMK-1 by western blotting. As a positive and negative controls, we examined the effect of $v h p-1$ RNAi and sek-1 RNAi. RNAi against sek-1 resulted in decreased phosphorylation of PMK-1/p38 without affecting total PMK-1 levels. RNAi against vhp-1 increased phospho-p38/PMK-1 levels without affecting total PMK-1 levels. RNAi treatment was begun at the $L 3$ developmental stage and worms were collected 2 days later. 
DAF-16 target genes
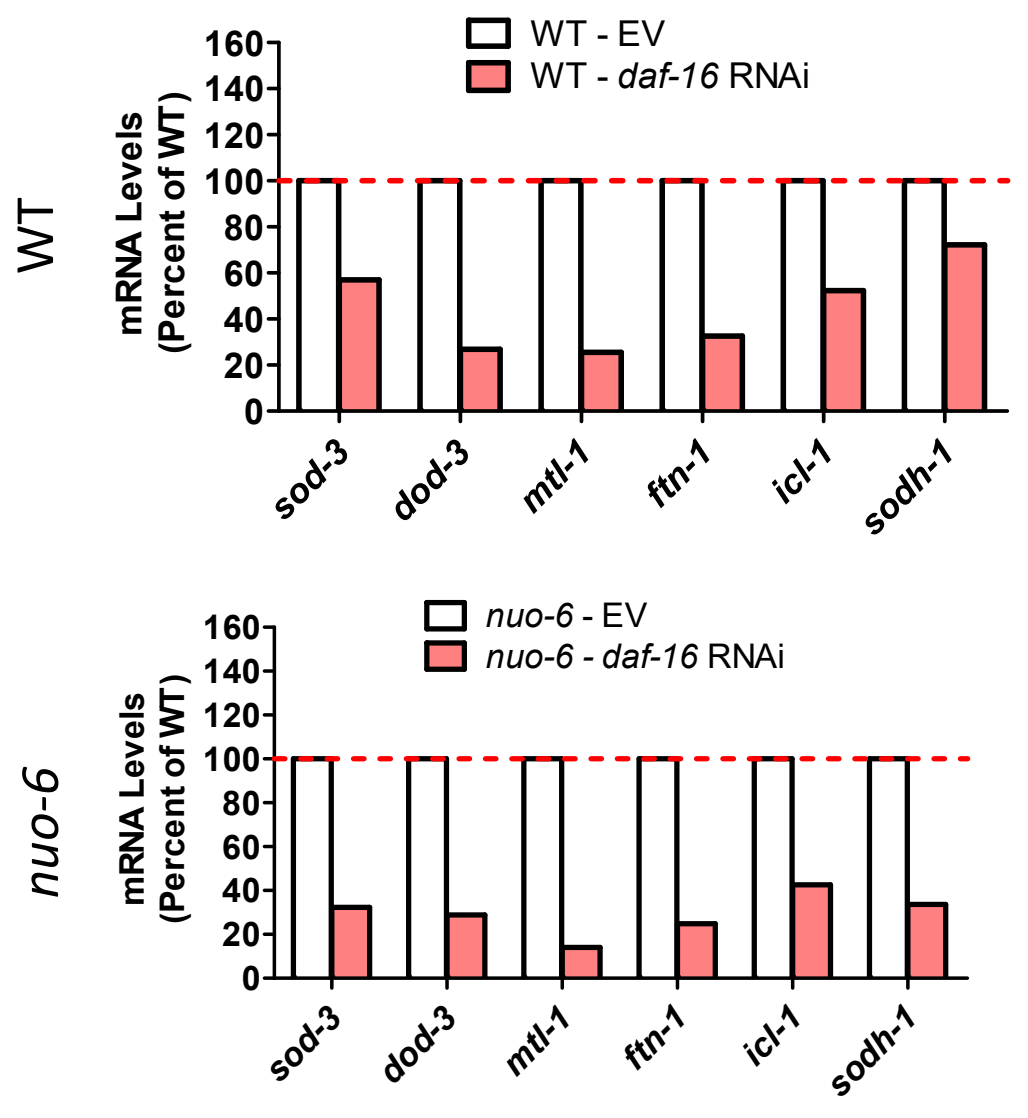

Innate immunity genes
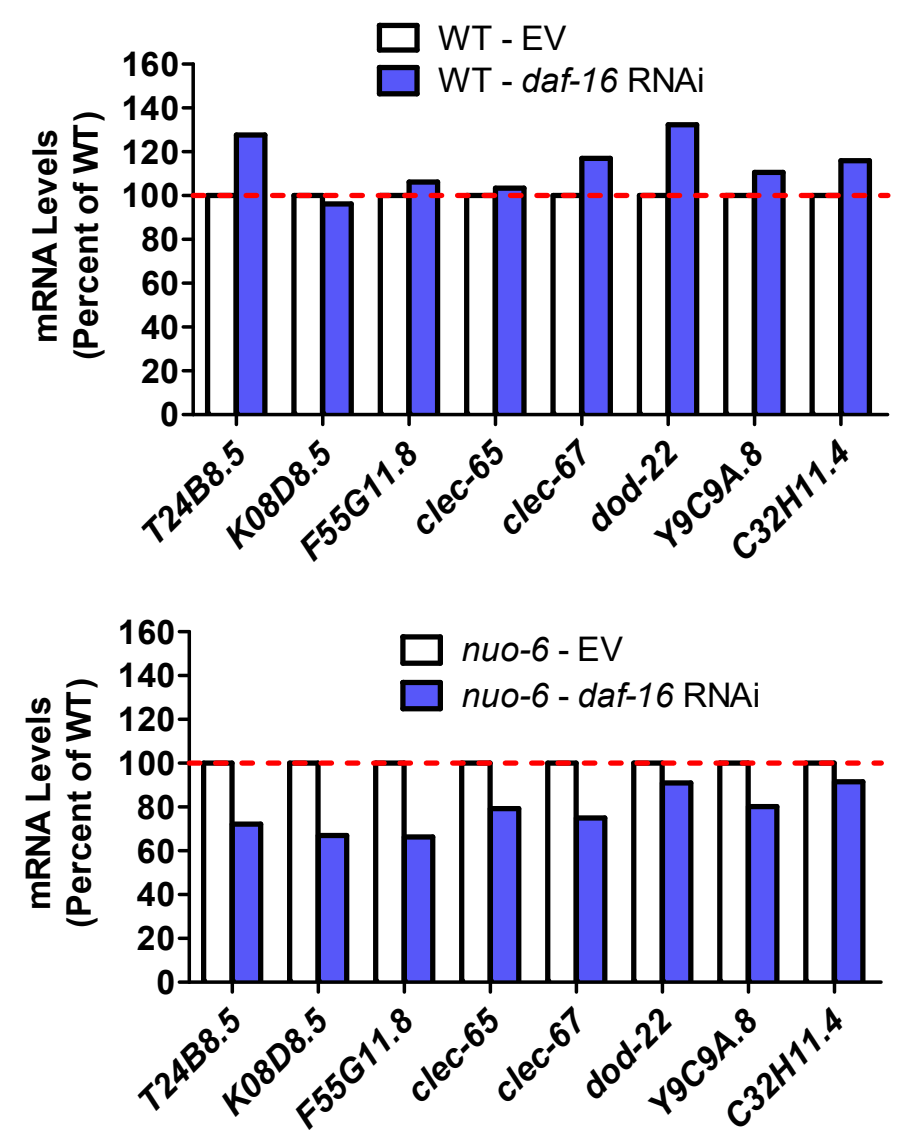

Figure S11. DAF-16 is not required for expression of innate immune signaling pathway target genes. daf-16 expression was knocked down using RNAi beginning at the L4 stage of the parental generation. While daf-16 RNAi effectively decreased the expression of DAF-16 target genes (b, sod-3, dod-3, mtl-1, ftn-1, icl-1, sodh-1) in both wild-type and nuo- 6 mutants, it did not markedly affect the expression of any of the innate immunity genes (c, T24B8.5, K08D8.5, F55G11.8, clec-65, clec-67, dod-22, Y9C9A.8 and C32H11.4). This suggests that DAF-16 is not required for expression of innate immune signaling pathway target genes in wild-type worms and nuo- 6 mutants. RNA was isolated from six biological replicates at the young adult stage of the experimental generation. RNA from the six biological replicates was pooled for RNA sequencing. 

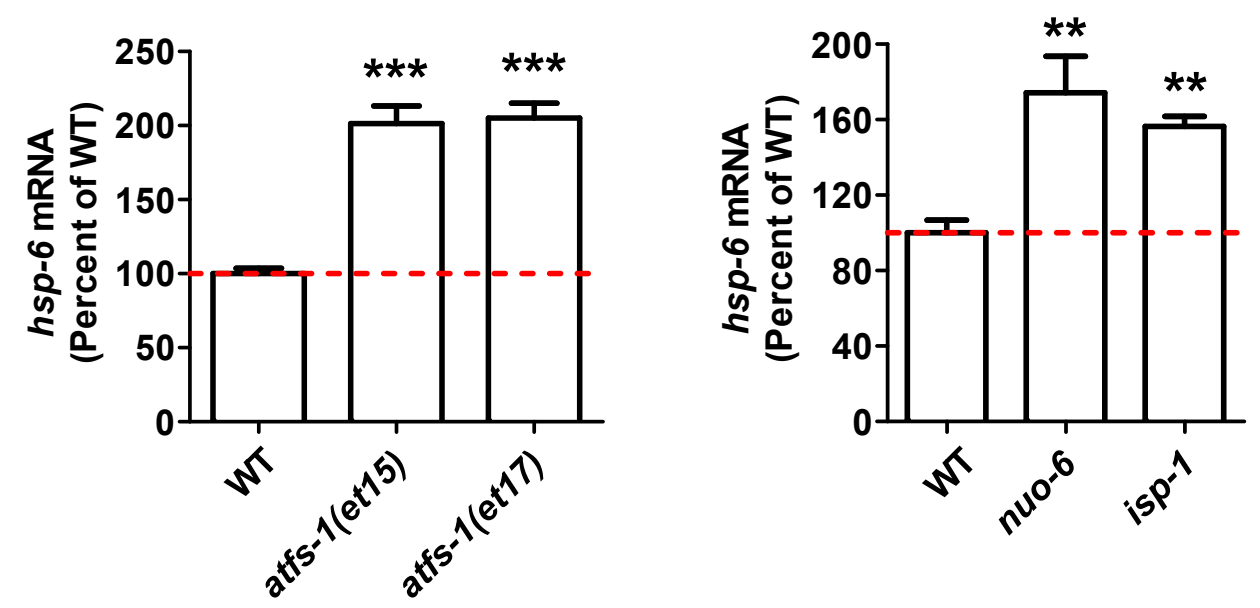

Figure S12. $\boldsymbol{h s p}$-6 levels are increased in constitutively active atfs-1 mutants. Gene expression changes were determined by RNA sequencing of six biological replicates of each genotype. Results represent counts per million (CPM) expressed as a percentage of wild-type. Error bars indicate SEM. ${ }^{* *} p<0.01,{ }^{* * *} p<0.001$. 


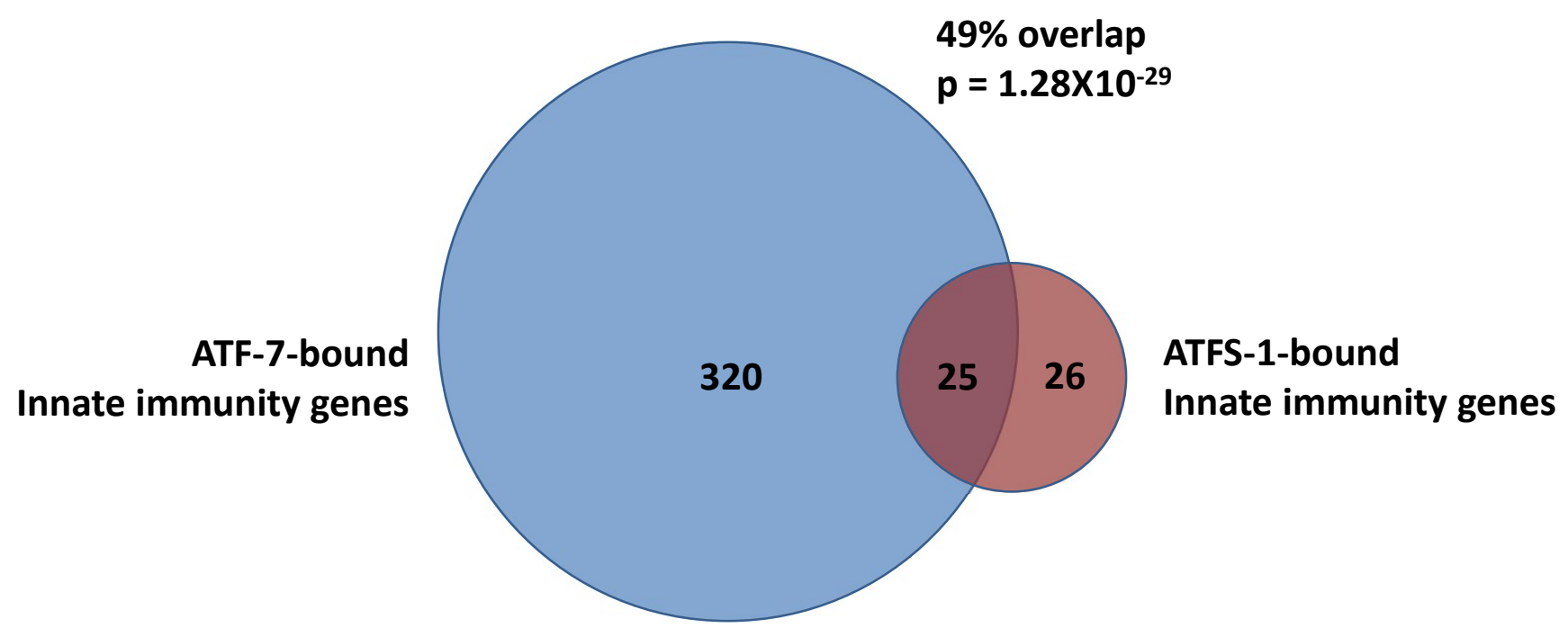

Figure S13. ATFS-1 and ATF-7 bind to the same genes involved in innate immunity. Innate immunity genes are defined as genes that are upregulated by a 4-hour exposure to PA14. This list was obtained from Fletcher et al., 2019. ATF-7-bound genes are genes bound by ATF-7 after exposure to PA14 from a CHIP-seq experiment conducted by Fletcher et al., 2019. ATFS-1-bound genes are genes bound by ATFS-1 after mitochondrial stress resulting from RNAi against spg-7 as determined by a CHIP-seq experiment conducted by Nargund et al., 2015. ATF-7 was found to be bound to 345 innate immunity genes after exposure to PA14. ATFS-1 was found to be bound to 51 innate immunity genes after exposure to mitochondrial stress. Of these 51 genes, 25 were found to be in common with ATF-7-bound innate immunity genes. This highly significant overlap $\left(p=1.28 \times 10^{-29}\right)$ clearly demonstrates that ATF-7 and ATFS-1 can bind to and regulate the same innate immunity genes. See supplemental Table 2 for the complete gene lists. 


\section{Phospho-p38}

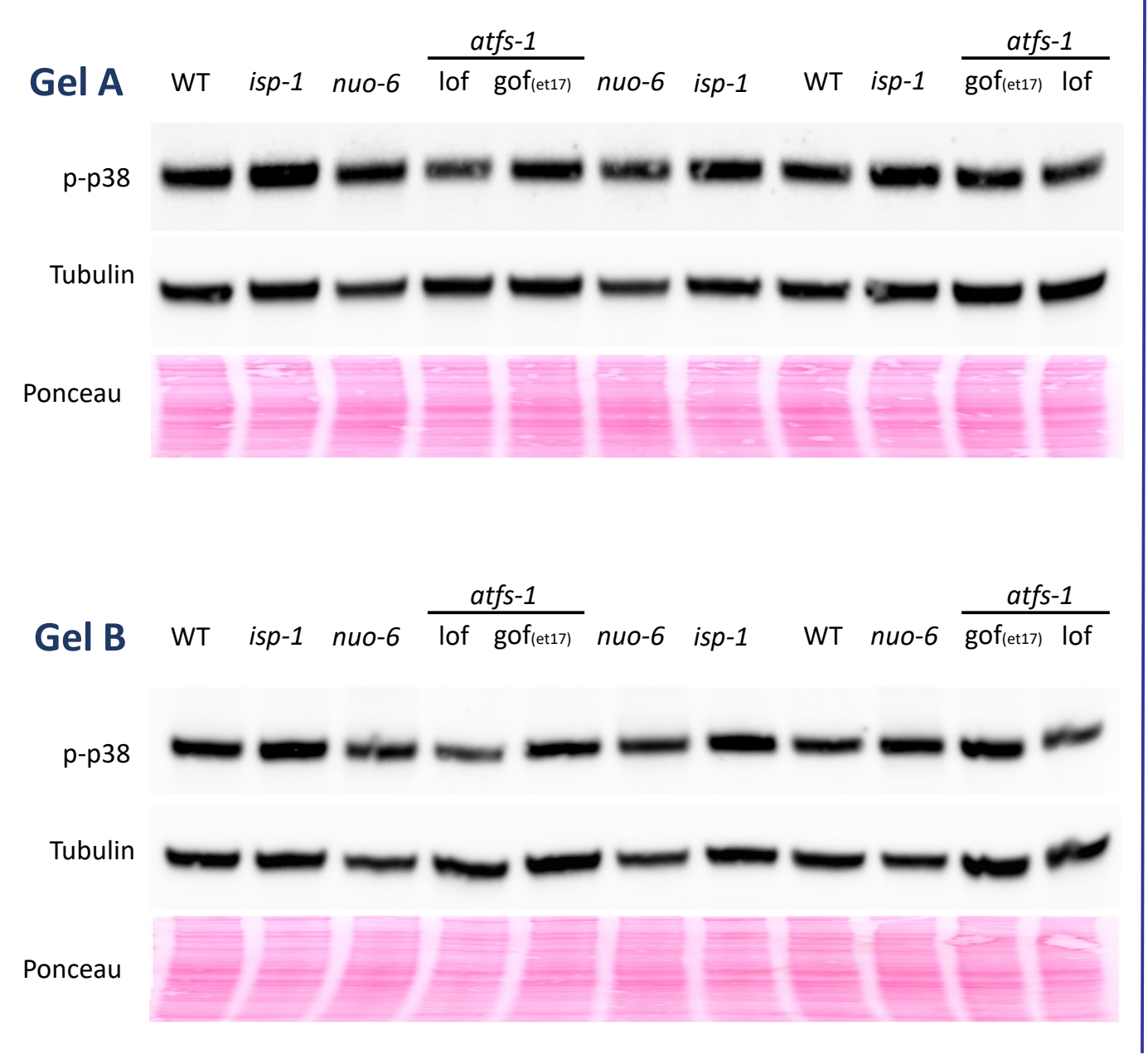

\section{p38/PMK-1}

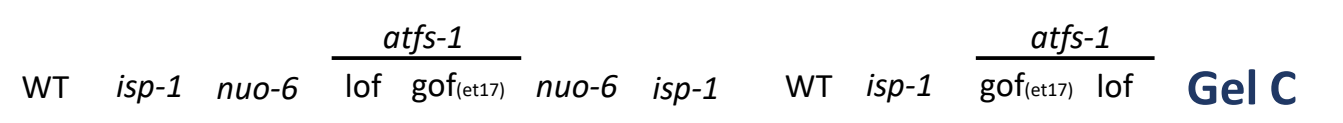

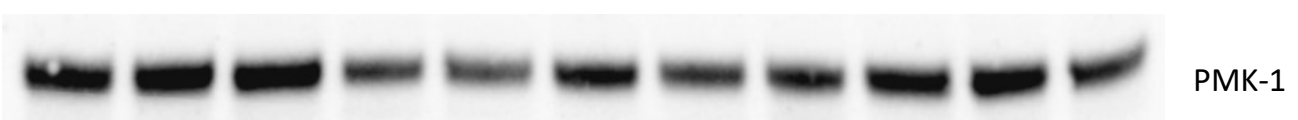

r

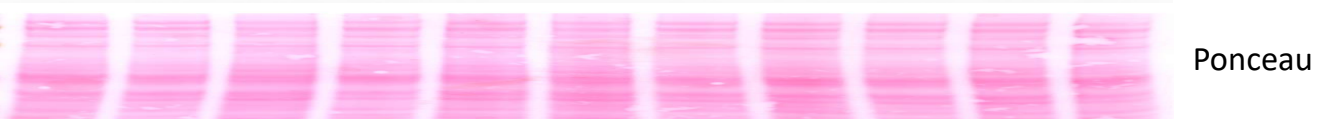

WT isp-1 nuo-6 $\frac{\text { atfs-1 }}{\text { lof gof (et17) }}$ nuo-6 isp-1 WT nuo-6 $\frac{\text { atfs-1 }}{\text { gof(et17) lof }}$ Gel D

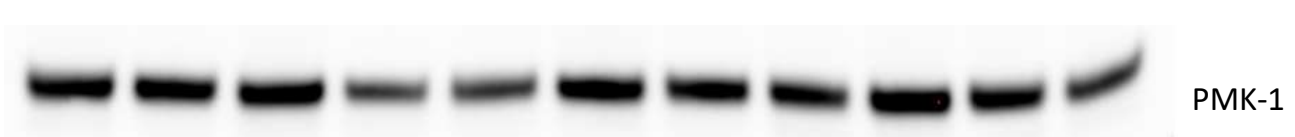

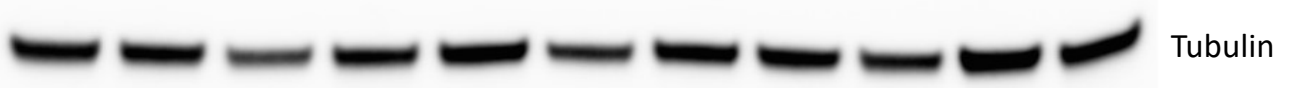
Ponceau 